\title{
Selecting the best unbalanced repeated measures model
}

\author{
Guillermo Vallejo • M. Paula Fernández • \\ Pablo E. Livacic-Rojas • Ellián Tuero-Herrero
}

Published online: 7 December 2010

(C) Psychonomic Society, Inc. 2010

\begin{abstract}
This study examined the performance of selection criteria available in the major statistical packages for both mean model and covariance structure. Unbalanced designs due to missing data involving both a moderate and large number of repeated measurements and varying total sample sizes were investigated. The study also investigated the impact of using different estimation strategies for information criteria, the impact of different adjustments for calculating the criteria, and the impact of different distribution shapes. Overall, we found that the ability of consistent criteria in any of the their examined forms to select the correct model was superior under simple covariance patterns than under complex covariance patterns, and vice versa for the efficient criteria. The simulation studies covered in this paper also revealed that, regardless of method of estimation used, the consistent criteria based on number of subjects were more effective than the consistent criteria based on total number of observations, and vice versa for the efficient criteria. Furthermore, results indicated that, given a dataset with
\end{abstract}

This paper was accepted for publication in Behavior Research Methods on October 25, 2010.

G. Vallejo $(\triangle) \cdot$ M. P. Fernández

Department of Psychology, University of Oviedo,

Plaza de Benito Feijóo, s/n 33003,

Oviedo, Spain

e-mail: gvallejo@uniovi.es

P. E. Livacic-Rojas

Department of Psychology, University of Santiago of Chile,

Avenida Ecuador 3650, Estación Central,

Santiago, Chile

E. Tuero-Herrero

Department of Psychology University of Oviedo,

Plaza de Benito Feijóo, s/n 33003,

Oviedo, Spain missing values, the efficient criteria were more affected than the consistent criteria by the lack of normality.

Keywords Information criteria - Longitudinal data . Maximum likelihood $\cdot$ Missing at random $\cdot$ Model selection $\cdot$ Restricted likelihood

\section{Introduction}

Repeated measures data arise when an outcome variable of interest is measured repeatedly for each individual in the study over a period of time. A key characteristic of repeated measures is the missing data problem. In many applications of psychology in the real world; missing values are inevitable, often taking the dropout form (i.e., a subject has observations up to a time point and none after that). Little and Rubin (2002) defined three types of missingness mechanisms for dropouts: missing completely at random (MCAR), missing at random (MAR), and missing not at random (MNAR). A dropout process is said to be MCAR when the missing data are independent both of observed and unobserved outcomes of the variable being analyzed (e.g., missing data occurring in follow-ups made after the treatment phase has ended), and said to be MAR if, conditional on the observed data (e.g., previous performance, lack of efficacy of assigned treatment or other subject characteristics), the missingness is independent of the unobserved data. A dropout process that is neither MCAR nor MAR is termed MNAR. Although in reality it is virtually impossible to properly specify the missingness mechanism, several studies, including those of Verbeke and Molenberghs (2000), Little and Rubin (2002), and DeSouza, Legedza, and Sankoh (2009), have concluded that MAR is a realistic mechanism for most 
practical applications because the study dropouts are frequently related to previous outcomes in the study.

Another characteristic feature of repeated measures studies is that observations within the same subject may be correlated, and for this reason, methods that account for the correlation among responses are often used. The widely used models include linear mixed effects models described by Laird and Ware (1982) and regression models with structured covariance proposed by Jennrich and Schluchter (1986).

In order to improve the quality of the inferences obtained with these approaches (i.e., to obtain valid and powerful tests of the fixed-effects parameter), it is important to choose a model with an appropriate mean and covariance structure (Littell, Pendergast, \& Natarajan, 2000; Fitzmaurice, Laird, \& Ware, 2004). When an appropriate model is selected, the precision and accuracy of parameter estimates improve, particularly when missing data are present. However, it is not always easy to find the model generating the data, because there are multiple candidate models for the same sample evidence (Claeskens \& Hjort, 2008).

Several general statistical software packages are available for the analysis of these models, including R/S-Plus, SAS, SPSS (now known as PASW), and STATA. Among them, SAS is the most widely used statistical package (Feng, Zhou, Zhang, \& Zhang, 2009). It is very useful for fitting different covariance patterns and uses various criteria to select the best fitting model. When two or more models are nested within each other, the likelihood ratio test can be used to discriminate between them. When models are not nested, information criteria are the most-used tool. An important advantage of using model selection criteria is that they can be used for nested and non-nested comparisons.

A variety of information criteria have been proposed for modeling longitudinal data with correlated errors. Selection tools such as the Akaike Information Criteria (AIC; Akaike, 1974), the corrected AIC (AICc; Hurvich \& Tsai, 1989), the Bayesian Information Criteria (BIC; Schwarz, 1978), the consistent AIC (CAIC; Bozdogan, 1987), and the HannanQuinn Information Criteria (HQIC; Hannan \& Quinn, 1979), are computed automatically when using Proc Mixed in SAS. The mixed command in SPSS/PASW provides four criteria (HQIC is not available), while the lme() function in $\mathrm{R} /$ Splus and $\mathrm{xtmixed}$ command in STATA only provide the AIC and BIC. Other software packages, such as HLM and MLwiN, do not provide any information criteria for selecting the most parsimonious correct model.

The performance of information criteria when interest lies in mixed model selection has been examined empirically under three different scenarios: (a) with respect to their ability to select the correct mean model given a particular covariance structure (Gurka, 2006; Wang \& Schaalje, 2009), (b) with respect to their ability to select the correct covariance structure when the mean model is known (Ferron, Dailey, \& Yi, 2002; Gomez, Schaalje, \& Fellingham, 2005; Gurka, 2006; Keselman, Algina, Kowalchuk, \& Wolfinger, 1998; Vallejo, Ato, \& Valdés, 2008), and (c) with respect to their ability to simultaneously select the correct mean and covariance structure (Gurka, 2006). All the studies cited compared the effectiveness of the AIC and BIC. Additionally, Gurka (2006) also compared the performance of the AICc and CAIC, and Vallejo et al. (2008) the performance of the HQIC. Although no consensus exists over what constitutes a good criterion for selecting the best model, given that the performance of the criteria depended on the conditions investigated, the results indicate that the selection improved as the sample size increased and the complexity of model decreased.

Among the studies mentioned above, Gurka's (2006) study is particularly relevant, since it offers a complete comparative review of performance criteria. Several findings from this study should be highlighted. First, the criteria based on the restricted/residual maximum likelihood (REML) log-likelihood function selected the true mean structure as well as or better than the criteria based on the maximum likelihood (ML) log-likelihood function, which contradicts the contention that REML-based information criteria are not appropriate for selecting the mean structure of the mixed model (Littell, Milliken, Stroup, Wolfinger, \& Schabenberger, 2006; Molenberghs \& Verbeke, 2001; Singer \& Willet, 2003). Second, the performance of information criteria in selecting the proper mean structure depended on the version of the REML function used. Third, the study provided valuable information with respect to discrepancies in the formulas for calculating the information criteria. It should be pointed out, however, that this simulation study was based on very simple scenarios (e.g., providing candidate models with few alternatives or using highly parsimonious covariance patterns to generate datasets). In words of Gurka (2006, p. 25) "the discussed simulation was admittedly limited, as a more sophisticated covariance model selection evaluation is worth an article by itself".

Therefore, the purpose of this article is to provide further insight into information criteria obtained using SAS's Proc Mixed when realistic sample sizes and more complex covariance models are used, both to generate incomplete data and compare the performance of criteria. A further contribution of this article is to provide some numerical evidence of the performance of information criteria in selecting the model generating the data when the normality assumption is violated. Although real data rarely conform to normality and missing data are common in longitudinal studies (Schafer \& Graham, 2002), these topics have not been thoroughly considered. Because most of the information criteria are derived using asymptotic normal theory, 
this study allowed their assessment under conditions that are often the norm rather than the exception in longitudinal data.

\section{Model and notation}

Let $y_{i j k}$ represent the response for the $i$ th subject $\left(i=1, \ldots, n_{j}\right)$, from the $j$ th group $(j=1, \ldots, g)$ at the $k$ th time point $\left(k=1, \ldots t_{i}\right)$. Also, let $n=\sum_{j}^{g} n_{j}$ denote the total number of subjects enrolled in the study and $m=\sum_{i}^{n} t_{i}$ denote the total number of observations in the dataset. The subscript $i$ means that no assumption of the complete data is being made.

In the context of repeated measures data, assume the data arise from the covariance pattern regression model

$\mathbf{y}_{i}=\mathbf{X}_{i} \boldsymbol{\beta}+\mathbf{e}_{i}$,

where $\mathbf{y}_{i}$ is a $\left(t_{i} \times 1\right)$ ector of observations for $i$ th subject, $\mathbf{X}_{i}$ is a $\left(t_{i} \times 1\right)$ matrix of known explanatory variables for $i$ th subject with rank $p, \beta$ is a $(p \times 1)$ vector of unknown regression parameters, and $\mathrm{e}_{i}$ is an $\left(t_{i} \times 1\right)$ error vector. For the $(i, j)$ th subject, it is assumed that the vector $\mathbf{e}_{i}$ is normally distributed with zero mean and covariance matrix $\boldsymbol{\Sigma}_{i}$ which is a function of a $q$-dimensional vector of unknown parameters $\theta$. Jennrich and Schluchter (1986) and Littell et al. (2006) consider several possible forms for $\Sigma_{i}$

Both ML and REML estimation are commonly used to obtain estimators for the parameters of model (1). Building on the normality assumption of the $\mathbf{e}_{i}$, minus 2 times the log-likelihood function based on the full data, apart from a constant, is

$$
\begin{aligned}
-2 l_{M L}(\boldsymbol{\beta}, \boldsymbol{\theta})= & \sum_{i=1}^{n} \log \left|\boldsymbol{\Sigma}_{i}\right| \\
& +\sum_{i=1}^{n}\left(\mathbf{y}_{i}-\mathbf{X}_{i} \boldsymbol{\beta}\right)^{\prime} \boldsymbol{\Sigma}_{i}^{-1}\left(\mathbf{y}_{i}-\mathbf{X}_{i} \boldsymbol{\beta}\right) .
\end{aligned}
$$

where $\widehat{\boldsymbol{\beta}}=\sum_{i=1}^{n}\left(\mathbf{X}_{i}^{\prime} \widehat{\boldsymbol{\Sigma}}_{i}^{-1} \mathbf{X}_{i}\right)^{-1} \sum_{i=1}^{n}\left(\mathbf{X}_{i}^{\prime} \widehat{\boldsymbol{\Sigma}}_{i}^{-1} \mathbf{y}_{i}\right)$ is the generalized least squares (GLS) estimator of vector $\beta$, assuming $\widehat{\Sigma}_{i}(\boldsymbol{\theta})$ is known. In practice, the ML estimators must be found from the data available by solving the Hessian matrix and score function using an iterative procedure. For details see Jennrich and Schluchter (1986) and Lindstrom and Bates (1988).

The ML estimators of $\beta$ and $\theta$ have desirable large sample properties. However, in finite samples, the ML estimator $\theta$ is biased. To circumvent this problem, Harville (1974) recommended the use of REML originally developed by Patterson and Thompson (1971) as a method of estimating variance components. Minus 2 times the restricted log-likelihood function, apart from a constant, takes the form

$$
\begin{aligned}
-2 l_{\text {REML }}(\boldsymbol{\theta})= & \sum_{i=1}^{n} \log \left|\boldsymbol{\Sigma}_{i}\right| \\
& +\sum_{i=1}^{n}\left(\mathbf{y}_{i}-\mathbf{X}_{i} \widehat{\boldsymbol{\beta}}\right)^{\prime} \boldsymbol{\Sigma}_{i}^{-1}\left(\mathbf{y}_{i}-\mathbf{X}_{i} \widehat{\boldsymbol{\beta}}\right) \\
& +\log \left|\sum_{i=1}^{n} \mathbf{X}_{i}^{\prime} \boldsymbol{\Sigma}_{i}^{-1} \mathbf{X}_{i}\right|-\log \left|\sum_{i=1}^{n} \mathbf{X}_{i}^{\prime} \mathbf{X}_{i}\right|
\end{aligned}
$$

where $\widehat{\beta}$ is the GLS estimator. This is identical to the REML function given by Harville (1974) and Cooper and Thompson (1977), among others.

When discussing model selection criteria, it is natural to ask: should one use ML or REML? The major software mixed models fitting procedures, such as R/Splus lme(), SAS Proc Mixed, SPSS/PASW mixed, and Stata xtmixed, allow a choice between both methods of estimation, with the default option usually being REML. For a further discussion of this problem, the reader is referred to West, Welch, and Galecki (2007) and McCulloch, Searle, and Neuhaus (2008). It should be pointed out, however, that many current mixed model- fitting procedures, such as SAS's Proc Mixed (SAS, 2008), omit the last term on the right side of (3) in the computation of the REML likelihood, which could impact the computing of an information criterion for comparing models having different mean structures. In fact, Gurka (2006) suggested using the REML function which has the term $\log \left|\sum_{i}^{n} \mathbf{X}_{i}^{\prime} \mathbf{X}_{i}\right|$, denoted as $l_{R E M L_{1}}$, for the consistent criteria in model selection, and using the REML function without the term $\log \left|\sum_{i}^{n} \mathbf{X}_{i}^{\prime} \mathbf{X}_{i}\right|$, denoted as $l_{R E M L_{2}}$, for the efficient criteria.

\section{Information criteria for model selection}

There has been extensive research in model selection criteria over the last decade (see Azari, Li, \& Tsai, 2006; Bozdogan, 2000; Jiang \& Rao, 2003; Kitagawa \& Konishi, 2010; Shang \& Cavanaugh, 2008, among others). Many of these developments have focused on using intensive computing techniques and on imposing a penalty on the likelihood that not only is related to sample size but to the dimension of estimated parameters $\widehat{\beta}$ and $\widehat{\theta}$ (e.g., with the use of the Fisher information matrix or generalized degrees of freedom). Despite their practical importance, they are not implemented in the widely used software programs, so they were not the focus of our attention. As indicated earlier, the purpose of this paper is to investigate the performance of information criteria currently available in statistical pack- 
ages for choosing a model with an appropriate mean and covariance structure, instead of examining the effectiveness of new proposed criteria.

When applying model selection criteria to obtain the best linear mixed model, one has to decide on the likelihood and correct number of parameters to use. In the linear mixed model the interest is either in the unknown regression (fixed effects) parameters, which are assumed to be shared by all individuals, or in the subject-specific deviation from the population estimates (random effects), which are assumed to be unique to a particular individual. For example, suppose we are testing the efficacy of a new therapy to reduce anxiety disorders. When the focus is on the fixed effects we want to know whether the average rate of change over time is different between the two groups, however, when the focus is on the random effects we want to know how individual profiles change over time in each group.

In the fixed-effects focus, the random effect is a device for modeling the correlation of the responses for $i$ th subject, and the model is often referred to as the marginal model with correlated errors or the population-averaged model without random effect (Gurka \& Edwards, 2008). Then, the problem is closely related to a regression model selection problem with correlated errors, and use of the marginal model does not involve defining information criteria different from those derived in the context of linear regression models. When the random effects are themselves of interest, then the conditional or hierarchical model should be used, and the conventional information criteria may be inappropriate (Liang, Wu, \& Zou, 2008; Vaida \& Blanchard, 2005).

In this study, all considered criteria have a form that consists of two basic elements. According to Lee and Ghosh (2009), one term measures the goodness-of-fit (loglikelihood) of a model and the other term penalizes the loglikelihood for the model complexity. In the following subsections, we describe the information criteria family examined in this paper, which are summarized in Table 1.

\section{Akaike's Information Criteria (AIC)}

Akaike (1974) suggested that the Kullback-Leibler (K-L) discrepancy measure provides a natural criterion for ordering candidate data models. The basic idea behind this criterion is to find within a predefined family of candidate models the one that minimizes the information loss occurring when the fitted candidate model is used as an approximation of the true model. The AIC in "smaller-isbetter" form is defined as

$A I C=-2 l_{M L}(\mathbf{y} \mid \widehat{\boldsymbol{\beta}}, \widehat{\theta})+2 s$

where $l_{M L}(\mathbf{y} \mid \widehat{\boldsymbol{\beta}}, \widehat{\theta})$ is the maximized log-likelihood function and $s=p+q$, with $p$ and $q$ representing the dimension of estimated parameters $\widehat{\beta}$ and $\widehat{\theta}$ under the given fitted candidate model. Here, the goodness-of-fit term, $-2 l_{M L}(\mathbf{y} \mid \widehat{\boldsymbol{\beta}}, \widehat{\theta})$, gauges how well the candidate model fits the data, and the
Table 1 True parameter values of the fixed effects used to generate the data
For purposes of comparison, the formulas listed in the table use $s=p+q . \mathrm{REML}_{1}$ is the residual maximum likelihood estimation including the last term of Eq. 3; REML $L_{2}$ is the residual maximum likelihood estimation excluding the last term of Eq. $3 ; n$ is the total number of subjects; $m$ is the total number of observations; $p$ is the rank of known design matrix; $q$ is the number of covariance parameters

\begin{tabular}{|c|c|c|c|}
\hline \multicolumn{2}{|c|}{ ML estimation } & \multicolumn{2}{|c|}{ REML estimation } \\
\hline Criteria & Definition & Criteria & Definition \\
\hline $\begin{array}{l}\mathrm{AIC} \\
\mathrm{AIC}_{2}\end{array}$ & $\begin{array}{l}\widehat{l}_{M L}+2 s \\
\widehat{l}_{R E M L_{2}}+2 s\end{array}$ & $\mathrm{AIC}_{1}$ & $\widehat{l}_{R E M L_{1}}+2 s$ \\
\hline $\mathrm{AICc}_{1}$ & $\widehat{l}_{M L}+2 s\left(\frac{m}{m-s-1}\right)$ & $\mathrm{AICc}_{1}$ & $\widehat{l}_{R E M L_{1}}+2 s\left(\frac{m-p}{m-s-1}\right)$ \\
\hline $\mathrm{AICc}_{2}$ & $\widehat{l}_{M L}+2 s\left(\frac{n}{n-s-1}\right)$ & $\begin{array}{l}\mathrm{AICc}_{2} \\
\mathrm{AICc}_{3} \\
\mathrm{AICc}_{4}\end{array}$ & $\begin{array}{l}\widehat{l}_{R E M L_{1}}+2 s\left(\frac{n}{n-s-1}\right) \\
\widehat{l}_{R E M L_{2}}+2 s\left(\frac{m-p}{m-s-1}\right) \\
\widehat{l}_{R E M L_{2}}+2 s\left(\frac{n}{n-s-1}\right)\end{array}$ \\
\hline $\mathrm{BIC}_{1}$ & $\widehat{l}_{M L}+s \log (m)$ & $\mathrm{BIC}_{1}$ & $\widehat{l}_{R E M L_{1}}+s \log (m-p)$ \\
\hline $\mathrm{BIC}_{2}$ & $\widehat{l}_{M L}+s \log (n)$ & $\begin{array}{l}\mathrm{BIC}_{2} \\
\mathrm{BIC}_{3} \\
\mathrm{BIC}_{4}\end{array}$ & $\begin{array}{l}\widehat{l}_{R E M L_{1}}+s \log (n) \\
\widehat{l}_{R E M L_{2}}+s \log (m-p) \\
\widehat{l}_{R E M L_{2}}+s \log (n)\end{array}$ \\
\hline $\mathrm{CAIC}_{1}$ & $\widehat{l}_{M L}+s[\log (m)+1]$ & $\mathrm{CAIC}_{1}$ & $\widehat{l}_{R E M L_{1}}+s[\log (m-p)+1]$ \\
\hline $\mathrm{CAIC}_{2}$ & $\widehat{l}_{M L}+s[\log (n)+1]$ & $\begin{array}{l}\mathrm{CAIC}_{2} \\
\mathrm{CAIC}_{3} \\
\mathrm{CAIC}_{4}\end{array}$ & $\begin{array}{l}\widehat{l}_{R E M L_{1}}+s[\log (n)+1] \\
\widehat{l}_{R E M L_{2}}+s[\log (m-p)+1] \\
\widehat{l}_{R E M L_{2}}+s[\log (n)+1]\end{array}$ \\
\hline $\mathrm{HQIC}_{1}$ & $\widehat{l}_{M L}+2 s \log [\log (m)]$ & $\mathrm{HQIC}_{1}$ & $\widehat{l}_{R E M L_{1}}+2 s \log [\log (m-p)]$ \\
\hline $\mathrm{HQIC}_{2}$ & $\widehat{l}_{M L}+2 s \log [\log (n)]$ & $\begin{array}{l}\mathrm{HQIC}_{2} \\
\mathrm{HQIC}_{3} \\
\mathrm{HQIC}_{4}\end{array}$ & $\begin{array}{l}\widehat{l}_{R E M L_{1}}+2 s \log [\log (n)] \\
\widehat{l}_{R E M L_{2}}+2 s \log [\log (m-p)] \\
\widehat{l}_{R E M L_{2}}+2 s \log [\log (n)]\end{array}$ \\
\hline
\end{tabular}


penalty term, $2 s$, measures the complexity that compensates for bias in the lack of fit. When REML estimation is used, $l_{M L}(\mathbf{y} \mid \widehat{\boldsymbol{\beta}}, \widehat{\theta})$ in (4) is replaced by the maximized REML loglikelihood $l_{R E M L}(\mathbf{y} \mid \widehat{\theta})$. Therefore, the number of parameters in (4) should be $s=q$, as used by Proc Mixed in SAS and mixed command in SPSS/PASW. However, xtmixed command in STATA and $l m e()$ function in R/Splus retain $s=p+q$, but for comparing between two models with the same fixed effects, this does not make any difference.

\section{Corrected Akaike's Information Criteria (AICc)}

The AIC provides us with an approximately unbiased estimator of the expected K-L discrepancy in settings where the sample size is large and the dimension of the model is comparatively small. However, in settings in which the sample size is small, it is known that the AIC is biased (Burnham \& Anderson, 2002). To account for this potential source of bias, Hurvich and Tsai (1989), based on the initial work of Sugiura (1978), proposed an efficient criterion, the corrected AIC (AICc), that outperforms the AIC in small samples, while converging with the AIC in larger samples. The AICc in "smaller-is-better" form is defined as

$A I C c=-2 l_{M L}(\mathbf{y} \mid \widehat{\boldsymbol{\beta}}, \widehat{\theta})+2 s\left(\frac{m^{*}}{m^{*}-s-1}\right)$

where $s=p+q$ and $m^{*}$ is the total number of observations $(m)$, as used by Proc Mixed in SAS and mixed command in SPSS/PASW. When REML estimation is used, $l_{M L}(\mathbf{y} \mid \widehat{\boldsymbol{\beta}}, \widehat{\theta})$ in (5) is replaced by the maximized REML $\log$-likelihood $l_{R E M L}(\mathbf{y} \mid \hat{\theta})$. Thus, the number of parameters in (5) should be $s=q$ and $m=m-p$ given that REML is based on $m-p$ observations. The AICc is sometimes defined where $m^{*}$ is the number of subjects $(n)$, under both ML and REML.

\section{Bayesian Information Criteria (BIC)}

Along with the AIC, another well-known and widely used tool in statistical model selection is the BIC. The criterion was derived by Schwarz (1978) from Bayesian theory as the solution to the identification problem. Unlike the AIC, which is designed to find the best approximating model to the unknown true data-generating model, the BIC is designed to find the most probable model given the data. The BIC in "smaller-is-better" form is defined as

$B I C=-2 l_{M L}(\mathbf{y} \mid \widehat{\boldsymbol{\beta}}, \widehat{\theta})+s \log \left(m^{*}\right)$

where $s=p+q$. An issue in using the BIC is that $m^{*}$ represents $n$, as used by SAS Proc Mixed or $m$, as used by
$\mathrm{R} /$ Splus lme(), Stata xtmixed, and SPSS/PASW mixed. When REML estimation is used, $l_{M L}(\mathbf{y} \mid \widehat{\boldsymbol{\beta}}, \widehat{\theta})$ in (6) is replaced by the maximized REML log-likelihood $l_{\text {REML }}(\mathbf{y} \mid \widehat{\theta})$. In the case of REML, $s=q$ and $m^{*}=n$ in SAS Proc Mixed, and $s=q$ and $m=m-p$ in SPSS/PASW mixed procedure, while $s=p+q$ and $m=m-p$ in R/Splus lme() and STATA xtmixed, respectively.

\section{Consistent Akaike's Information Criterion (CAIC)}

It is known that the AIC tends to select overly complex models, and does not produce an asymptotically consistent estimate of model order. Bozdogan (1987) proposed another consistent version of the AIC that takes sample size into account, and corrects the liberal tendency of the AIC by penalizing over-parameterization. Just like the BIC, the CAIC points to the right model with probability of unity as the sample size increases. The CAIC in "smaller-isbetter" form is defined as

$C A I C=-2 l_{M L}(\mathbf{y} \mid \widehat{\boldsymbol{\beta}}, \widehat{\theta})+s\left[\log \left(m^{*}\right)+1\right]$

where $s=p+q$. As with the BIC, an issue in using the CAIC is that $m^{*}$ represents $n$, as used by SAS Proc Mixed or $m$, as used by SPSS/PASW mixed. When REML estimation is used, $l_{M L}(\mathbf{y} \mid \widehat{\boldsymbol{\beta}}, \widehat{\theta})$ in (7) is replaced by the maximized REML log-likelihood $l_{\text {REML }}(\mathbf{y} \mid \widehat{\theta})$. In the case of REML, $s=q$ and $m^{*}=n$ in SAS Proc Mixed, and $s=q$ and $m=m-p$ in SPSS/PASW mixed procedure. R/Splus lme() and Stata xtmixed do not compute this criterion.

\section{Hannan-Quinn's Information Criterion (HQIC)}

Since the AIC tends to identify values of $p$ and $q$ that are too large while the BIC tends to underestimate the optimal model, Hannan and Quinn (1979) developed a consistent criterion that slows the changes occurring in the penalty term upon varying the sample size. The HQIC in "smalleris-better" form is defined as

$H Q I C=-2 l_{M L}(\mathbf{y} \mid \widehat{\boldsymbol{\beta}}, \widehat{\theta})+2 s \log \left[\log \left(m^{*}\right)\right]$

where $s=p+q$ and $m^{*}=m$. When REML estimation is used, $l_{M L}(\mathbf{y} \mid \widehat{\boldsymbol{\beta}}, \widehat{\theta})$ in (8) is replaced by the maximized REML log-likelihood $l_{\text {REML }}(\mathbf{y} \mid \widehat{\boldsymbol{\theta}})$. In the case of REML, $s=q$ and the reported value of $m^{*}$ in SAS Proc Mixed is $m-p \mathrm{R} /$ Splus $\operatorname{lme}()$, Stata $x$ tmixed, and SPSS/PASW mixed do not compute this criterion. 


\section{Simulation study}

To establish the relative merits of information criteria in selecting the best regression model with patterned covariance structure, we conducted a simulation study using a completely randomized design in which $n$ subjects were randomly distributed in $g$ treatment groups with $t$ equally spaced measurements from each subject. As indicated by Algina and Keselman (2004), the design used in this work has appeared in the literature under a variety of names (i.e., group randomized trials design, randomized parallel-groups design, split-plot repeated measures design), however, in all situations the hypothesis of interest is whether there are differential rates of change over time. To investigate the performance of these criteria in a situation with missing data we manipulated the following variables: types of mean and covariance structures, number of repeated measurements per experimental unit, total sample size, population distribution shape, and estimation methods used to determine the appropriateness of criteria. Variables concerning the number of groups, pattern of missing data, and homogeneity of covariance matrices were held constant in this study.

\section{Study variables}

To compare the procedures, two different mean models were used to generate data: (1) a full model with a common intercept, a dummy variable indicating membership in one of two groups, a continuous covariate that took equally spaced values in each group, and an additional group $x$ slope interaction; and (2) a reduced model with a common intercept, a linear trend, and an additional group covariate. Specifically, we used the following two equations to generate data for the response $\mathrm{y}_{i j k}$ measured at time $k$ for subject $i$ in group $j$ :

$y_{i j k}=\beta_{0}+\beta_{1} \operatorname{Trt}_{i j}+\beta_{2} \operatorname{Time}_{i k}+\beta_{3} \operatorname{Trt}_{i j} \times \operatorname{Time}_{i k}+e_{i j k}$,

$y_{i j k}=\beta_{0}+\beta_{1} \operatorname{Trt}_{i j}+\beta_{2}$ Time $_{i k}+e_{i j k}$.

where $\operatorname{Trt}_{i j}$ denotes an indicator variable for subject $i$ in treatment group $j$ (i.e., $\operatorname{Tr} t=0$ for placebo control; $\operatorname{Tr} t=1$ for active treatment), and Time $_{i k}$ was coded 0 to $t$ time points.

Each of the two regression models was paired with one of the following covariance structures: (a) first-order autoregressive covariance pattern (AR), (b) first-order autoregressive covariance pattern with variance heterogeneity within subjects (ARH), (c) Toeplitz covariance pattern with variance heterogeneity within subjects (TOEPH), and (d) unstructured covariance pattern (UN). The former structure is extensively used for modeling covariance in longitudinal data. However, the latter are potentially more realistic because the assumption of constant variance across time is relaxed. Because published research reports contain no large empirical studies in which experienced judges show the covariance patterns likely to be encountered in practice, it is difficult to known how typical or pervasive the chosen covariance structures are. Thus, though the structures considered in the simulation might be regarded as arbitrarily chosen they nonetheless coincide with conditions previously investigated by several authors, including Keselman, Algina et al. (1998), Gomez et al. (2005), and Vallejo et al. (2008), and they are broad enough to represent data sets that may be encountered in applied settings. In all cases, population values for covariance matrices were chosen so that the variances at each time point were relatively small at the beginning of the study, the traces were identical, and the deviations from sphericity were similar. As indicated above, covariance structures were assumed to be the same for all subjects in the two treatment groups $\left(\Sigma_{i j}=\Sigma\right)$ Details on these structures when the number of repeated measurements was eight $(t=8)$ are in Table 2.

A two-group parallel design containing either $t=4$ or $t=8$ repeated measures per subject was considered. In behavioral sciences research, more than four observations per subject are not typical; in fact, the mode is equal to 4 , at least according to the survey results provided by Kowalchuk, Lix, and Keselman (1996), while a large number of repeated observations per subject would not be unusual in clinical trials. We initially planned to investigate $t=10$, however, preliminary simulations indicated that using SAS Proc Mixed took an inordinate amount of time when $t=10$. It is doubtful that this change substantially affected the results.

For each value of $t$, three total sample sizes were considered, $n=30\left(n_{1}=n_{2}=15\right), n=60\left(n_{1}=n_{2}=30\right)$, and $n=120\left(n_{1}=n_{2}=60\right)$. These sample sizes were selected, in part, because they are typical of what is encountered in applied research (see. e.g., Keselman, Huberty et al. 1998). The small sample size yielded a priori statistical power of approximately $40 \%$ for the time main effect and the interaction between time and group at the 5\% Type I error rate. For the same design effects, the moderate and large sample sizes provided power values of approximately $60 \%$ and $80 \%$, respectively. Since our work assumed there were missing data with repeated measures due to dropout, analytic power calculations may not be readily available (Kleinman \& Horton, 2010). For this reason, the values of betas given in Table 3 for the $.40, .60$, and .80 target powers were calculated using numerical techniques and SAS Proc Mixed. The selected set of regression coefficients under the full model represents a situation in which the rate of change is greater in the treatment group 
Table 2 Parameter values of the covariance patterns used to generate the data

(a) First-Order Autoregressive (AR), 2 parameters

$\boldsymbol{\Sigma}_{1}=\operatorname{Cov}\left(e_{i j k}, e_{i j k^{\prime}}\right)=\sigma^{2}$ if $k=k^{\prime}$ and $\sigma^{2} \rho^{\left|k-k^{\prime}\right|}$ if $k \neq k^{\prime}, \sigma^{2}=136.5 ; \rho=.8$;

(b) Heterogeneous First-Order Autoregressive (ARH), $t+1$ parameters

$$
\begin{aligned}
& \boldsymbol{\Sigma}_{2}=\operatorname{Cov}\left(e_{i j k}, e_{i j k^{\prime}}\right)=\sigma_{k} \sigma_{k^{\prime}} \text { if } k=k^{\prime} \text { and } \sigma_{\mathrm{k}} \sigma_{\mathrm{k}^{\prime}} \rho^{\left|\mathrm{k}-\mathrm{k}^{\prime}\right|} \text { if } \mathrm{k} \neq \mathrm{k} \\
& \sigma_{1}^{2}=47 ; \sigma_{2}^{2}=48.5 ; \sigma_{3}^{2}=62 ; \sigma_{4}^{2}=87.5 ; \sigma_{5}^{2}=125 ; \sigma_{6}^{2}=174.5 ; \sigma_{7}^{2}=236 ; \sigma_{8}^{2}=309.5 ; \rho=.8 ;
\end{aligned}
$$

(c) Heterogeneous Toeplitz (TOEPH), $2 t-1$ parameters

$$
\begin{aligned}
& \boldsymbol{\Sigma}_{3}=\operatorname{Cov}\left(e_{i j k}, e_{i j k^{\prime}}\right)=\sigma_{k} \sigma_{k^{\prime}} \text { if } k=k^{\prime} \text { and } \sigma_{\mathrm{k}} \sigma_{\mathrm{k}^{\prime}} \rho_{\left|\mathrm{k}-\mathrm{k}^{\prime}\right|} \text { if } \mathrm{k} \neq \mathrm{k} \\
& \sigma_{1}^{2}=47 ; \sigma_{2}^{2}=48.5 ; \sigma_{3}^{2}=62 ; \sigma_{4}^{2}=87.5 ; \sigma_{5}^{2}=125 ; \sigma_{6}^{2}=174.5 ; \sigma_{7}^{2}=236 ; \sigma_{8}^{2}=309.5 ; \\
& \rho_{1}=.8 ; \rho_{2}=.7 ; \rho_{3}=.6 ; \rho_{4}=.5 ; \rho_{5}=.4 ; \rho_{6}=.3 ; \rho_{7}=.2 .
\end{aligned}
$$

(d) Unstructured (UN), $t(t+1) / 2$ parameters

$$
\begin{aligned}
\Sigma_{4} & =\operatorname{Cov}\left(e_{i j k}, e_{i j k^{\prime}}\right)=\sigma_{k}^{2} \text { if } k=k \text { and } \sigma_{k k^{\prime}} \text { if } k \neq k^{\prime}, \\
\sigma_{1}^{2} & =47 ; \sigma_{2}^{2}=48.5 ; \sigma_{3}^{2}=62 ; \sigma_{4}^{2}=87.5 ; \sigma_{5}^{2}=125 ; \sigma_{6}^{2}=174.5 ; \sigma_{7}^{2}=236 ; \sigma_{8}^{2}=309.5 ; \\
\sigma_{12} & =43.0 ; \sigma_{13}=45.9 ; \sigma_{14}=51.3 ; \sigma_{15}=57.5 ; \sigma_{16}=63.4 ; \sigma_{17}=68.5 ; \sigma_{18}=72.4 ; \\
\sigma_{23} & =46.6 ; \sigma_{24}=52.1 ; \sigma_{25}=58.4 ; \sigma_{26}=64.4 ; \sigma_{27}=69.5 ; \sigma_{28}=73.5 ; \sigma_{34}=58.9 ; \\
\sigma_{35} & =66.0 ; \sigma_{36}=72.8 ; \sigma_{37}=78.6 ; \sigma_{38}=83.1 ; \sigma_{45}=78.4 ; \sigma_{46}=86.5 ; \sigma_{47}=93.4 ; \\
\sigma_{48} & =98.7 ; \sigma_{56}=103.4 ; \sigma_{57}=111.6 ; \sigma_{58}=1118 ; \sigma_{67}=131.9 ; \sigma_{68}=139.4 ; \sigma_{78}=162.2 .
\end{aligned}
$$

\begin{tabular}{|c|c|c|c|c|c|c|c|c|c|c|c|c|c|c|}
\hline & \multicolumn{8}{|c|}{ Full model } & \multicolumn{6}{|c|}{ Reduced model } \\
\hline & \multicolumn{4}{|c|}{$t=4$} & \multicolumn{4}{|c|}{$t=8$} & \multicolumn{3}{|c|}{$t=4^{2}$} & \multicolumn{3}{|c|}{$t=8$} \\
\hline & $\beta_{0}$ & $\beta_{1}$ & $\beta_{2}$ & $\beta_{3}$ & $\beta_{0}$ & $\beta_{1}$ & $\beta_{2}$ & $\beta_{3}$ & $\beta_{0}$ & $\beta_{1}$ & $\beta_{2}$ & $\beta_{0}$ & $\beta_{1}$ & $\beta_{2}$ \\
\hline \multicolumn{15}{|c|}{$1-\beta \approx .40\left(n_{1}=15 ; n_{2}=15\right)$} \\
\hline AR & 25 & 0.00 & -1.45 & -2.15 & 25 & 0.00 & -1.20 & -1.60 & 25 & 5.45 & -1.45 & 25 & 4.95 & -1.20 \\
\hline $\mathrm{ARH}$ & 25 & 0.00 & -1.40 & -2.00 & 25 & 0.00 & -1.20 & -1.55 & 25 & 4.85 & -1.40 & 25 & 4.20 & -1.20 \\
\hline TOEPH & 25 & 0.00 & -1.30 & -1.40 & 25 & 0.00 & -1.10 & -1.40 & 25 & 4.57 & -1.30 & 25 & 4.20 & -1.10 \\
\hline UN & 25 & 0.00 & -1.50 & -2.15 & 25 & 0.00 & -0.60 & -0.75 & 25 & 4.15 & -1.50 & 25 & 3.45 & -0.60 \\
\hline \multicolumn{15}{|c|}{$1-\beta \approx .60\left(n_{1}=30 ; n_{2}=30\right)$} \\
\hline $\mathrm{AR}$ & 25 & 0.00 & -1.35 & -1.90 & 25 & 0.00 & -1.00 & -1.50 & 25 & 5.10 & -1.35 & 25 & 4.65 & -1.00 \\
\hline $\mathrm{ARH}$ & 25 & 0.00 & -1.30 & -1.80 & 25 & 0.00 & -1.00 & -1.45 & 25 & 4.35 & -1.30 & 25 & 3.95 & -1.00 \\
\hline TOEPH & 25 & 0.00 & -1.10 & -1.60 & 25 & 0.00 & -0.90 & -1.30 & 25 & 4.20 & -1.10 & 25 & 4.00 & -0.90 \\
\hline UN & 25 & 0.00 & -1.30 & -1.80 & 25 & 0.00 & -0.50 & -0.70 & 25 & 3.75 & -1.30 & 25 & 3.00 & -0.50 \\
\hline \multicolumn{15}{|c|}{$1-\beta \approx .80\left(n_{1}=60 ; n_{2}=60\right)$} \\
\hline AR & 25 & 0.00 & -1.20 & -1.65 & 25 & 0.00 & -0.90 & -1.25 & 25 & 4.45 & -1.20 & 25 & 3.85 & -0.90 \\
\hline $\mathrm{ARH}$ & 25 & 0.00 & -1.15 & -1.60 & 25 & 0.00 & -0.90 & -1.25 & 25 & 3.70 & -1.15 & 25 & 3.55 & -0.90 \\
\hline TOEPH & 25 & 0.00 & -1.00 & -1.40 & 25 & 0.00 & -0.80 & -1.05 & 25 & 3.55 & -1.00 & 25 & 3.50 & -0.80 \\
\hline UN & 25 & 0.00 & -1.10 & -1.50 & 25 & 0.00 & -0.40 & -0.60 & 25 & 3.35 & -1.10 & 25 & 2.65 & -0.40 \\
\hline
\end{tabular}

The upper left $4 \times 4$ matrix was used when the number of repeated measures was four. For $t=4, \sigma=61.25$ in the case (a)

than in the control group, but at the first time point the groups do no differ. On the other hand, the selected set of regression coefficients under the reduced model represents a situation in the which the patterns of change in the mean response over time is the same in both groups, but the population mean response profiles are parallel. In other words, the regression coefficients of these two models were selected to obtain an ordinal interaction and equal slopes in

Table 3 True parameter values of the fixed effects used to generate the data 
the different groups. Both scenarios are of high scientific interest for investigating treatment effects in a repeated measures design.

Two population distributions were considered using the methods described in the subsequent section. The multivariate normal distribution with univariate skew $\left(\gamma_{1}\right)$ and kurtosis $\left(\gamma_{2}\right)$ equal to zero $\left(\gamma_{1}=\gamma_{2}=0\right)$ was the first. A moderately skewed distribution with shape parameters equivalent to those of an exponential distribution $\left(\gamma_{1}=2\right.$ and $\gamma_{2}=6$ ) was the second. Level of non-normality examined was representative of that often encountered in educational and psychological research (Micceri, 1989).

Finally, the performance of the AIC, AICc, BIC, CAIC, and the HQIC for each of 96 data types was evaluated first under ML and then under REML estimation, using both the REML ${ }_{1}$ and REML ${ }_{2}$ function, again using the terminology in Gurka (2006). Therefore, the simulation plan used a $2^{4} \times 3 \times 4$ factorial design, with 192 different cells or conditions.

\section{Data generation}

In each treatment group, Gaussian continuous longitudinal data were simulated using the method of Ripley (1987). This procedure involves the following two steps:

1) Generate pseudorandom observation vectors $\mathbf{z}_{i j}$ with $E$ $\left(\mathbf{z}_{i j}\right)=\mathbf{0}$ and $\operatorname{Cov}\left(\mathbf{z}_{i j}\right)=\mathbf{I}$ from a $t$-variate normal distribution, where $\mathbf{I}$ is the identity matrix. These vectors were obtained using the RANNOR function in SAS.

2) Create complete data sets $\mathbf{y}_{i j}$ by multiplying the vector $\mathbf{z}_{i j}$ by the Cholesky decomposition $\mathbf{L}_{l}$, that is, $\mathbf{y}_{i j}=$ $\beta_{j}+\mathbf{L}_{l} \mathbf{z}_{i j}$, where $\mathbf{y}_{i j}$ is a vector of length $t$ for the $(i, j)$ th subject, $\beta j$ is a $p$-dimensional vector containing the population fixed effects, and $\mathbf{L}_{l}$ is a lower triangular matrix of dimension $t$ satisfying $\boldsymbol{\Sigma}_{l}=\mathbf{L}_{l} \mathbf{L}_{l}^{\prime}, l=1, \ldots 4$, as presented in Table 2 .

Then, longitudinal data with a monotone missing pattern were generated according to a specific type of MAR dropout model, namely, after the first time point, nonresponse at a given time point is a function of the response at the previous occasion, which was always observed. Specifically, if the value of the dependent variable at occasion $k$ was lower than a cutoff value $\lambda$ (observed values of outcome variable measure at occasion $k-1$ ) and if $U_{k}$ (a uniform random variable) was less than a probability determined for $\gamma$ (an expected proportion of missing data), then the subject dropped out at the occasion $k$ and the subsequent occasions. The constants $\lambda$ and $\gamma$ were chosen to yield approximate average cumulative dropout rates of 0 , 10,20 , and $30 \%$ in the $t=4$ condition, and average cumulative dropout rates of $0,5,10,15,20,25,35$, and $50 \%$ in the $t=8$ condition. These two dropout rates were assumed to be constant across time points and distributed evenly between the two groups.

Multivariate non-normal data were generated through the power method developed by Fleishman (1978) and extended to the multivariate situation by Vale and Maurelli (1983). The steps used to implement this procedure can be summarized as follows:

1) Calculate a weight vector $\mathbf{w}=[a b c d]^{\prime}$ with the desired skew and kurtosis for each distribution using Fleishman's power transformation method.

2) Compute an appropriate intermediate correlation matrix $\mathbf{R}_{l}$ by solving for all possible pairs of repeated measurements with the following third-order polynomial equation: $R_{X_{k} X_{k^{\prime}}}=\rho_{Z_{k} Z_{k^{\prime}}}\left(b^{2}+6 b d+9 d^{2}\right)+\rho_{Z_{k} Z_{k^{\prime}}}^{2} 2 c^{2}+\rho_{Z_{k} Z_{k^{\prime}}}^{3} 6 d^{2}$, where $\rho_{Z_{k} Z_{k^{\prime}}}$ is the correlation coefficient between any two standard normal variables and $X_{k}\left(=a+b Z_{k}+\right.$ $\left.c Z_{k}^{2}+d Z_{k}^{3}\right)$ and $X_{k^{\prime}}\left(=a+b Z_{k^{\prime}}+c Z_{k^{\prime}}^{2}+d Z_{k^{\prime}}^{3}\right)$ are the two correlated non-normal variables.

3) Factorize the matrix $\mathbf{R}_{\boldsymbol{l}}$ to generate a vector of multivariate normal random variates with the prescribed $\rho_{Z_{k} Z_{k^{\prime}}}$, that is, $\mathbf{x}_{i j}=\mathbf{M}_{l} \mathbf{z}_{i j}$, where $\mathbf{x}_{i j}$ denotes the vector of transformed variates with $E\left(\mathbf{x}_{i j}\right)=\mathbf{0}$ and $\operatorname{Cov}\left(\mathbf{x}_{i j}\right)=\mathbf{R}_{l}$, and $\mathbf{M}_{l}$ is the lower triangular matrix in the Cholesky decomposition with the property $\mathbf{R}_{l}=$ $\mathbf{M}_{l} \mathbf{M}_{l}^{\prime}$.

4) Transform the generated multivariate normal variates to the desired distributional shape and the desired population fixed effects and variances, that is, $\mathbf{y}_{i j}=$ $\boldsymbol{\beta}_{j}+\mathbf{D}_{l}\left(\mathbf{X}_{i j}^{\bullet} \mathbf{w}\right)$, where $\mathbf{D}_{l}$ is a diagonal matrix containing the standard deviations of covariance matrix $\boldsymbol{\Sigma}_{l}$ defined in Table 2 and $\mathbf{X}_{i j}^{\bullet}=\left[\mathbf{1}_{K} \mathbf{x}_{i j} \mathbf{x}_{i j}^{2} \mathbf{x}_{i j}^{3}\right]$.

Simulated data were generated under each of the 192 conditions manipulated in the study, and a range of 36 models was fitted to each simulated condition. In particular, the two models referred to above for generating data, and a null model, were combined with twelve different covariance structures. These structures included: compound symmetry (CS), heterogeneous compound symmetry (CSH), linear random coefficients (RCL), first-order autoregressive (AR), heterogeneous first-order autoregressive (ARH), first-order factor analytic (FA), first-order autoregressive moving-average (ARMA), Huynh-Feldt (HF), Toeplitz (TOEP), heterogeneous Toeplitz (TOEPH), firstorder antedependence (ANTE), and unstructured (UN). For all 36 possible models, an optimal model was selected computing the five information criteria and their examined variations. As shown in Table 1, nine used ML estimation and eighteen used REML estimation.

This process was repeated 5,000 times for each condition using a SAS macro. To evaluate the performance of these 
information criteria we recorded the percentage of times each one of these criteria detected the model generating the data. When the estimation failed to converge, it was assumed that none of the criteria selected that model.

\section{Simulation results}

In order to assess the performance of the consistent criteria (BIC, CAIC, and HQIC) and their efficient counterparts (AIC and $\mathrm{AICc}$ ) in selecting the best regression model with patterned covariance, we computed the percentage of times these criteria selected the model generating the data under ML and under REML, using both REML estimation with the term $\log \left|\sum_{i}^{n} \mathbf{X}_{i}^{\prime} \mathbf{X}_{i}\right|$ (denoted as $\mathrm{REML}_{1}$ ) as well as without it (denoted as $\mathrm{REML}_{2}$ ). For comparison, we also considered the variations of five criteria based on the total sample size $(n)$ and the total number of observations $(m)$. Tables 4, 5, 6 and 7 display the numerical results obtained from Monte Carlo simulations. Tables 4 and 5 report the results when data were both multivariate normal and nonnormal (i.e., exponential-type data) in form and the full model was used to generate the data, whereas Tables 6 and 7 present the results when data were both multivariate normal and non-normal in form and the reduced model was adopted.

\section{Performances associated with normal data for full model}

The percentages of correct decisions when the data were normally distributed and the full model was adopted are found in Table 4. These results can be summarized as follows:

1) Simulation results show that no one criterion selected the model generating the data consistently nor performed uniformly better than the others. Averaging across the covariance structures, total sample size, number of repeated measurements, and methods of estimation, the overall success was $56.4 \%$ for the AIC, $48.3 \%$ for the AICc, $39.1 \%$ for the BIC, $32.6 \%$ for the CAIC, and $52.1 \%$ for the HQIC.

2) In general, the performance of the consistent criteria (BIC, CAIC, and HQIC) was superior to efficient criteria (AIC and $\mathrm{AICc}$ ) for relatively simple covariance patterns (i.e., AR and ARH), whereas efficient criteria were superior for more complex covariance patterns (i.e., TOEPH and UN). It was also found that the performance of the AIC and AICc was fairly comparable, however, the AICC did not perform better than the AIC when sample sizes were moderate to small. On the other hand, the CAIC gave results almost identical to those of the BIC, although overall the BIC performed slightly better than the CAIC.

3) All versions of the five criteria performed better for larger numbers of subjects and performed much better for designs in which the number of repeated measurements was large. For moderate and large sample sizes, it is very apparent that the effect of sample size was greater when the length of repeated measures was short. In particular, the performance at $n=120$ and $t=8$ was slightly better than at $n=60$ and $t=8$, better than at $n=120$ and $t=4$, and substantially better than at $n=60$ and $t=4$.

4) When comparing the consistent criteria based on $n$ and the consistent criteria based on $m$, the former led to a considerably larger percentage of correct decisions, regardless of whether ML or REML estimation was used. On the other hand, the performance of the AICc did not show this behavior. In fact, the simulation results pointed towards the use of $m$ under REML and $n$ under ML.

5) Finally, it is important to highlight that the five criteria and their examined variations performed better under REML than under ML estimation. The average differences between the two methods of estimation were approximately 9 percentage points for the efficient criteria and approximately 13 percentage points for the consistent criteria. It is also noteworthy that the ability of criteria to select the correct model was superior under $\mathrm{REML}_{1}$ than under REML $\mathrm{L}_{2}$. The average differences between the two versions of REML estimation were approximately nine percentage points for the efficient criteria and approximately 19 percentage points for the consistent criteria.

\section{Performances associated with non-normal data and full model}

The percentages of correct decisions when the data were obtained from a moderately skewed distribution and the full model was adopted are found in Table 5. These results can be summarized as follows:

1) The pattern of results found under departures from normality was qualitatively similar to the pattern described for the normal distribution; however, from a quantitative point of view the shape of the distribution substantially affected the percentage of correct decisions. Averaging across the covariance structures, total sample size, number of repeated measurements, and methods of estimation, the overall success was $35.2 \%$ for the AIC, $33.3 \%$ for the AICc, $32.7 \%$ for the BIC, $28.4 \%$ for the CAIC, and $39.5 \%$ for the HQIC. 
Table 4 Percentages of correct identifications for normal data and full model $(t=4,8)$

\begin{tabular}{|c|c|c|c|c|c|c|c|c|c|c|c|c|}
\hline \multicolumn{4}{|c|}{$n=30\left(n_{1}=n_{2}=15\right)$} & \multicolumn{4}{|c|}{$n=60\left(n_{1}=n_{2}=30\right)$} & \multicolumn{5}{|c|}{$n=120\left(n_{1}=n_{2}=60\right)$} \\
\hline $\mathrm{CP}_{1}$ & $\mathrm{CP}_{2}$ & $\mathrm{CP}_{3}$ & $\mathrm{CP}_{4}$ & $\mathrm{CP}_{1}$ & $\mathrm{CP}_{2}$ & $\mathrm{CP}_{3}$ & $\mathrm{CP}_{4}$ & $\mathrm{CP}_{1}$ & $\mathrm{CP}_{2}$ & $\mathrm{CP}_{3}$ & $\mathrm{CP}_{4}$ & \\
\hline \multicolumn{13}{|c|}{ REML estimation $(t=4)$} \\
\hline $\mathrm{AIC}_{1}$ & 57.8 & 35.2 & 18.7 & 14.5 & 67.1 & 51.6 & 31.9 & 24.2 & 66.9 & 70.0 & 65.9 & 42.5 \\
\hline $\mathrm{AIC}_{2}$ & 56.5 & 34.1 & 15.3 & 13.8 & 61.2 & 47.0 & 27.4 & 22.0 & 64.9 & 65.0 & 62.3 & 41.2 \\
\hline $\mathrm{AICc}_{1}$ & 63.7 & 33.5 & 11.2 & 9.1 & 69.1 & 53.7 & 25.5 & 20.9 & 69.9 & 71.7 & 60.4 & 41.6 \\
\hline $\mathrm{AICc}_{2}$ & 74.0 & 12.5 & 5.4 & 0.4 & 80.0 & 55.2 & 11.7 & 5.4 & 87.1 & 77.2 & 65.5 & 34.8 \\
\hline $\mathrm{AICc}_{3}$ & 61.6 & 26.8 & 12.9 & 10.6 & 62.8 & 48.5 & 26.4 & 19.6 & 67.1 & 66.5 & 61.8 & 40.1 \\
\hline $\mathrm{AICc}_{4}$ & 67.0 & 10.9 & 3.1 & 0.4 & 68.6 & 44.1 & 10.3 & 3.9 & 71.9 & 70.1 & 59.9 & 31.4 \\
\hline $\mathrm{HQIC}_{1}$ & 82.3 & 24.4 & 9.3 & 3.8 & 87.2 & 45.0 & 8.2 & 9.5 & 92.2 & 77.0 & 38.9 & 13.8 \\
\hline $\mathrm{HQIC}_{2}$ & 70.8 & 30.2 & 12.5 & 7.9 & 81.4 & 51.1 & 17.7 & 12.6 & 88.7 & 80.1 & 50.6 & 25.3 \\
\hline $\mathrm{HQIC}_{3}$ & 64.9 & 17.6 & 5.8 & 2.5 & 68.9 & 35.9 & 7.4 & 4.2 & 81.7 & 63.9 & 33.6 & 11.7 \\
\hline $\mathrm{HQIC}_{4}$ & 62.6 & 27.4 & 8.2 & 6.9 & 69.9 & 42.9 & 15.6 & 10.9 & 81.3 & 69.9 & 44.7 & 21.1 \\
\hline $\mathrm{BIC}_{1}$ & 87.4 & 9.2 & 0.3 & 0.1 & 93.2 & 20.7 & 0.7 & 0.2 & 97.3 & 51.5 & 7.6 & 1.5 \\
\hline $\mathrm{BIC}_{2}$ & 84.1 & 20.9 & 3.4 & 2.3 & 90.8 & 45.8 & 3.6 & 2.4 & 96.8 & 77.7 & 19.1 & 8.6 \\
\hline $\mathrm{BIC}_{3}$ & 51.6 & 5.5 & 0.2 & 0.0 & 56.1 & 11.7 & 0.3 & 0.1 & 69.8 & 40.7 & 4.8 & 1.6 \\
\hline $\mathrm{BIC}_{4}$ & 62.8 & 16.0 & 1.5 & 1.8 & 67.7 & 24.2 & 2.4 & 1.1 & 78.4 & 68.0 & 15.5 & 6.7 \\
\hline $\mathrm{CAIC}_{1}$ & 85.2 & 4.9 & 0.3 & 0.0 & 90.8 & 14.0 & 0.0 & 0.1 & 92.8 & 38.4 & 3.3 & 0.6 \\
\hline $\mathrm{CAIC}_{2}$ & 85.6 & 13.0 & 0.9 & 0.2 & 92.6 & 30.8 & 1.2 & 0.4 & 95.6 & 66.8 & 10.2 & 2.5 \\
\hline $\mathrm{CAIC}_{3}$ & 41.8 & 1.5 & 0.0 & 0.0 & 49.3 & 4.7 & 0.0 & 0.0 & 62.3 & 32.3 & 1.7 & 0.4 \\
\hline $\mathrm{CAIC}_{4}$ & 54.0 & 9.0 & 0.4 & 0.0 & 59.3 & 13.7 & 0.5 & 0.2 & 72.3 & 57.4 & 6.6 & 1.7 \\
\hline \multicolumn{13}{|c|}{ ML estimation $(t=4)$} \\
\hline AIC & 34.0 & 22.1 & 11.7 & 10.6 & 50.7 & 40.4 & 29.6 & 22.5 & 64.3 & 62.5 & 60.6 & 31.2 \\
\hline $\mathrm{AICc}_{1}$ & 40.8 & 12.2 & 5.3 & 0.0 & 58.0 & 36.1 & 12.7 & 3.5 & 69.6 & 66.1 & 55.7 & 19.0 \\
\hline $\mathrm{AICc}_{2}$ & 37.9 & 16.6 & 12.5 & 5.2 & 51.7 & 41.9 & 28.9 & 19.9 & 65.9 & 63.8 & 59.1 & 28.1 \\
\hline $\mathrm{HQIC}_{1}$ & 37.9 & 13.3 & 4.2 & 2.3 & 57.3 & 30.0 & 7.3 & 5.1 & 78.4 & 61.2 & 30.6 & 9.7 \\
\hline $\mathrm{HQIC}_{2}$ & 38.1 & 20.3 & 8.0 & 5.8 & 57.7 & 37.3 & 13.9 & 10.4 & 77.1 & 64.6 & 39.0 & 16.2 \\
\hline $\mathrm{BIC}_{1}$ & 31.2 & 9.1 & 0.3 & 0.2 & 44.2 & 12.4 & 0.5 & 0.2 & 66.7 & 31.3 & 3.6 & 0.8 \\
\hline $\mathrm{BIC}_{2}$ & 37.5 & 17.4 & 1.0 & 1.6 & 54.3 & 21.3 & 3.7 & 1.0 & 73.4 & 48.0 & 13.9 & 4.3 \\
\hline $\mathrm{CAIC}_{1}$ & 26.7 & 6.3 & 0.2 & 0.0 & 35.9 & 7.9 & 0.1 & 0.1 & 58.9 & 21.7 & 1.4 & 0.2 \\
\hline $\mathrm{CAIC}_{2}$ & 34.1 & 11.5 & 0.3 & 0.2 & 47.2 & 15.8 & 0.5 & 0.4 & 68.0 & 36.2 & 5.5 & 0.9 \\
\hline \multicolumn{13}{|c|}{ REML estimation $(t=8)$} \\
\hline $\mathrm{AIC}_{1}$ & 69.5 & 71.8 & 74.2 & 92.8 & 74.8 & 83.9 & 95.8 & 99.0 & 77.3 & 87.7 & 99.4 & 99.8 \\
\hline $\mathrm{AIC}_{2}$ & 60.8 & 56.9 & 61.0 & 64.1 & 64.7 & 69.8 & 76.4 & 66.1 & 73.4 & 82.7 & 92.8 & 88.1 \\
\hline $\mathrm{AICc}_{1}$ & 72.6 & 81.6 & 68.7 & 86.2 & 77.0 & 87.2 & 95.0 & 99.2 & 78.7 & 88.8 & 99.8 & 99.8 \\
\hline $\mathrm{AICc}_{2}$ & 0.0 & 0.2 & 0.0 & 15.0 & 82.7 & 94.6 & 73.7 & 0.0 & 84.3 & 97.1 & 99.8 & 99.9 \\
\hline $\mathrm{AICc}_{3}$ & 63.7 & 64.5 & 54.6 & 52.4 & 67.9 & 72.1 & 72.5 & 64.2 & 75.5 & 83.6 & 92.4 & 86.3 \\
\hline $\mathrm{AICc}_{4}$ & 0.0 & 0.2 & 0.0 & 5.4 & 72.4 & 66.6 & 38.5 & 0.0 & 80.4 & 89.0 & 88.5 & 72.9 \\
\hline $\mathrm{HQIC}_{1}$ & 86.9 & 92.4 & 34.2 & 45.5 & 92.2 & 97.3 & 77.8 & 99.0 & 93.2 & 99.6 & 99.2 & 99.9 \\
\hline $\mathrm{HQIC}_{2}$ & 79.6 & 85.3 & 63.0 & 90.4 & 88.6 & 96.8 & 91.3 & 99.7 & 91.7 & 99.0 & 99.8 & 99.9 \\
\hline $\mathrm{HQIC}_{3}$ & 57.3 & 60.8 & 26.9 & 25.3 & 65.2 & 66.4 & 55.0 & 50.6 & 88.0 & 82.6 & 80.6 & 76.0 \\
\hline $\mathrm{HQIC}_{4}$ & 64.8 & 63.9 & 48.6 & 54.0 & 66.5 & 70.8 & 61.6 & 57.3 & 82.0 & 87.8 & 86.4 & 81.0 \\
\hline $\mathrm{BIC}_{1}$ & 91.6 & 75.8 & 0.0 & 0.2 & 98.2 & 95.2 & 22.4 & 25.5 & 99.5 & 99.8 & 84.1 & 97.2 \\
\hline $\mathrm{BIC}_{2}$ & 86.8 & 92.6 & 34.8 & 44.8 & 94.8 & 96.6 & 69.4 & 97.6 & 96.3 & 99.9 & 98.0 & 99.8 \\
\hline $\mathrm{BIC}_{3}$ & 38.4 & 31.3 & 0.0 & 0.0 & 45.4 & 41.0 & 9.1 & 10.6 & 66.7 & 61.8 & 52.9 & 55.2 \\
\hline $\mathrm{BIC}_{4}$ & 57.1 & 60.4 & 26.8 & 25.0 & 60.9 & 65.5 & 40.0 & 45.0 & 77.4 & 78.5 & 72.0 & 70.1 \\
\hline $\mathrm{CAIC}_{1}$ & 88.8 & 59.5 & 0.0 & 0.7 & 96.3 & 94.6 & 9.0 & 3.8 & 97.1 & 99.0 & 69.5 & 92.0 \\
\hline
\end{tabular}


Table 4 (continued)

\begin{tabular}{|c|c|c|c|c|c|c|c|c|c|c|c|c|}
\hline \multicolumn{4}{|c|}{$n=30\left(n_{1}=n_{2}=15\right)$} & \multicolumn{4}{|c|}{$n=60\left(n_{1}=n_{2}=30\right)$} & \multicolumn{5}{|c|}{$n=120\left(n_{1}=n_{2}=60\right)$} \\
\hline $\mathrm{CP}_{1}$ & $\mathrm{CP}_{2}$ & $\mathrm{CP}_{3}$ & $\mathrm{CP}_{4}$ & $\mathrm{CP}_{1}$ & $\mathrm{CP}_{2}$ & $\mathrm{CP}_{3}$ & $\mathrm{CP}_{4}$ & $\mathrm{CP}_{1}$ & $\mathrm{CP}_{2}$ & $\mathrm{CP}_{3}$ & $\mathrm{CP}_{4}$ & \\
\hline $\mathrm{CAIC}_{2}$ & 90.0 & 89.0 & 16.9 & 5.2 & 96.6 & 98.0 & 47.7 & 71.4 & 98.0 & 99.7 & 94.1 & 99.6 \\
\hline $\mathrm{CAIC}_{3}$ & 31.2 & 21.4 & 0.0 & 0.2 & 37.4 & 32.6 & 2.8 & 1.8 & 59.3 & 54.4 & 37.5 & 47.2 \\
\hline $\mathrm{CAIC}_{4}$ & 47.6 & 47.2 & 9.1 & 2.4 & 54.6 & 52.9 & 20.4 & 27.2 & 72.0 & 70.8 & 63.2 & 63.4 \\
\hline \multicolumn{13}{|c|}{ ML estimation $(t=8)$} \\
\hline AIC & 40.5 & 43.9 & 45.6 & 64.8 & 57.1 & 60.3 & 69.0 & 75.8 & 70.7 & 80.6 & 95.7 & 91.6 \\
\hline $\mathrm{AICc}_{1}$ & 0.0 & 0.0 & 0.0 & 0.0 & 68.7 & 57.0 & 36.5 & 0.0 & 78.8 & 87.1 & 82.9 & 88.8 \\
\hline $\mathrm{AICc}_{2}$ & 46.2 & 49.4 & 39.3 & 52.1 & 64.4 & 62.1 & 67.7 & 72.8 & 74.3 & 81.8 & 95.3 & 91.4 \\
\hline $\mathrm{HQIC}_{1}$ & 43.7 & 45.5 & 13.6 & 30.6 & 70.8 & 52.9 & 42.3 & 58.8 & 92.6 & 81.5 & 86.3 & 81.8 \\
\hline $\mathrm{HQIC}_{2}$ & 48.6 & 49.2 & 35.9 & 58.1 & 71.6 & 60.0 & 58.4 & 67.6 & 91.1 & 85.6 & 90.4 & 86.3 \\
\hline $\mathrm{BIC}_{1}$ & 37.3 & 21.5 & 1.2 & 0.2 & 59.4 & 29.5 & 8.1 & 13.2 & 93.6 & 60.7 & 66.8 & 52.1 \\
\hline $\mathrm{BIC}_{2}$ & 53.6 & 45.6 & 13.7 & 30.1 & 69.8 & 48.0 & 33.9 & 52.1 & 97.4 & 74.9 & 80.3 & 74.4 \\
\hline $\mathrm{CAIC}_{1}$ & 26.0 & 13.0 & 0.4 & 0.0 & 52.7 & 23.6 & 3.2 & 2.5 & 85.5 & 53.7 & 60.8 & 38.8 \\
\hline $\mathrm{CAIC}_{2}$ & 38.0 & 34.1 & 3.9 & 11.0 & 63.2 & 37.9 & 19.3 & 36.0 & 94.6 & 67.1 & 76.0 & 64.5 \\
\hline
\end{tabular}

$\overline{\mathrm{CP}_{1}}=$ first-order autoregressive covariance pattern; $\mathrm{CP}_{2}=$ heterogeneous first-order autoregressive covariance pattern; $\mathrm{CP}_{3}=$ heterogeneous Toeplitz covariance pattern; $\mathrm{CP}_{4}=$ unstructured covariance pattern

Table 5 Percentages of correct identifications for non-normal data and full model $(t=4,8)$

\begin{tabular}{|c|c|c|c|c|c|c|c|c|c|c|c|c|}
\hline & \multicolumn{4}{|c|}{$n=30\left(n_{1}=n_{2}=15\right)$} & \multicolumn{4}{|c|}{$n=60\left(n_{1}=n_{2}=30\right)$} & \multicolumn{4}{|c|}{$n=120\left(n_{1}=n_{2}=60\right)$} \\
\hline & $\mathrm{CP}_{1}$ & $\mathrm{CP}_{2}$ & $\mathrm{CP}_{3}$ & $\mathrm{CP}_{4}$ & $\mathrm{CP}_{1}$ & $\mathrm{CP}_{2}$ & $\mathrm{CP}_{3}$ & $\mathrm{CP}_{4}$ & $\mathrm{CP}_{1}$ & $\mathrm{CP}_{2}$ & $\mathrm{CP}_{3}$ & $\mathrm{CP}_{4}$ \\
\hline \multicolumn{13}{|c|}{ REML estimation $(t=4)$} \\
\hline $\mathrm{AIC}_{1}$ & 21.5 & 31.8 & 22.0 & 23.2 & 19.5 & 37.6 & 34.2 & 30.6 & 18.5 & 42.8 & 54.2 & 44.7 \\
\hline $\mathrm{AIC}_{2}$ & 21.3 & 30.1 & 20.6 & 14.2 & 18.2 & 34.7 & 31.1 & 27.8 & 17.0 & 39.1 & 49.7 & 41.5 \\
\hline $\mathrm{AICc}_{1}$ & 26.3 & 35.7 & 19.7 & 15.3 & 21.9 & 40.4 & 34.0 & 25.2 & 19.5 & 44.2 & 55.3 & 44.8 \\
\hline $\mathrm{AICc}_{2}$ & 56.1 & 37.1 & 12.0 & 2.0 & 33.6 & 52.2 & 28.9 & 10.3 & 24.7 & 54.4 & 60.4 & 35.2 \\
\hline $\mathrm{AICc}_{3}$ & 26.0 & 33.3 & 18.5 & 14.5 & 20.6 & 36.9 & 31.1 & 23.7 & 18.1 & 40.9 & 54.5 & 37.9 \\
\hline $\mathrm{AICc}_{4}$ & 41.9 & 23.0 & 9.0 & 1.9 & 29.4 & 43.8 & 23.6 & 7.2 & 22.0 & 45.6 & 51.3 & 29.0 \\
\hline $\mathrm{HQIC}_{1}$ & 45.9 & 33.9 & 10.7 & 6.3 & 47.1 & 52.3 & 32.2 & 10.7 & 49.1 & 67.4 & 52.2 & 16.6 \\
\hline $\mathrm{HQIC}_{2}$ & 35.4 & 35.4 & 16.8 & 13.7 & 36.4 & 49.8 & 28.1 & 19.5 & 40.2 & 62.8 & 57.4 & 35.8 \\
\hline $\mathrm{HQIC}_{3}$ & 35.2 & 27.3 & 9.0 & 4.9 & 36.3 & 40.7 & 17.7 & 8.8 & 40.1 & 52.3 & 42.3 & 13.9 \\
\hline $\mathrm{HQIC}_{4}$ & 29.1 & 30.3 & 13.3 & 10.5 & 30.7 & 41.1 & 23.5 & 14.9 & 33.8 & 50.2 & 47.5 & 23.4 \\
\hline $\mathrm{BIC}_{1}$ & 63.5 & 30.2 & 4.9 & 0.5 & 68.3 & 49.3 & 5.7 & 1.1 & 72.0 & 67.7 & 20.6 & 3.3 \\
\hline $\mathrm{BIC}_{2}$ & 54.9 & 35.9 & 11.5 & 4.1 & 65.4 & 54.5 & 14.2 & 3.9 & 68.4 & 78.3 & 41.8 & 7.7 \\
\hline $\mathrm{BIC}_{3}$ & 36.2 & 35.5 & 3.0 & 0.3 & 40.1 & 25.7 & 3.6 & 0.7 & 45.2 & 41.4 & 15.1 & 1.6 \\
\hline $\mathrm{BIC}_{4}$ & 36.5 & 23.6 & 9.1 & 3.0 & 43.6 & 36.0 & 11.7 & 2.9 & 45.3 & 50.3 & 28.0 & 5.5 \\
\hline $\mathrm{CAIC}_{1}$ & 70.0 & 23.8 & 1.2 & 0.2 & 75.4 & 38.8 & 1.9 & 0.5 & 76.2 & 62.1 & 15.7 & 0.9 \\
\hline $\mathrm{CAIC}_{2}$ & 68.4 & 31.4 & 6.9 & 0.9 & 71.0 & 51.9 & 10.5 & 1.4 & 74.1 & 74.3 & 28.8 & 3.7 \\
\hline $\mathrm{CAIC}_{3}$ & 31.6 & 10.3 & 0.9 & 0.1 & 38.5 & 18.4 & 1.8 & 0.5 & 41.5 & 31.9 & 9.7 & 0.5 \\
\hline $\mathrm{CAIC}_{4}$ & 36.2 & 18.1 & 3.1 & 0.7 & 43.1 & 29.0 & 6.7 & 1.0 & 48.3 & 45.8 & 18.9 & 1.5 \\
\hline \multicolumn{13}{|c|}{ ML estimation $(t=4)$} \\
\hline AIC & 6.6 & 19.4 & 10.8 & 14.4 & 10.3 & 29.4 & 28.6 & 23.6 & 12.4 & 39.3 & 42.3 & 29.1 \\
\hline $\mathrm{AICc}_{1}$ & 12.1 & 14.3 & 2.9 & 0.2 & 24.9 & 34.1 & 20.8 & 7.8 & 23.9 & 45.4 & 44.5 & 27.8 \\
\hline $\mathrm{AICc}_{2}$ & 7.9 & 10.9 & 9.4 & 8.0 & 15.1 & 31.9 & 29.5 & 20.5 & 18.1 & 39.9 & 42.9 & 25.7 \\
\hline
\end{tabular}


Table 5 (continued)

\begin{tabular}{|c|c|c|c|c|c|c|c|c|c|c|c|c|}
\hline & \multicolumn{4}{|c|}{$n=30\left(n_{1}=n_{2}=15\right)$} & \multicolumn{4}{|c|}{$n=60\left(n_{1}=n_{2}=30\right)$} & \multicolumn{4}{|c|}{$n=120\left(n_{1}=n_{2}=60\right)$} \\
\hline & $\mathrm{CP}_{1}$ & $\mathrm{CP}_{2}$ & $\mathrm{CP}_{3}$ & $\mathrm{CP}_{4}$ & $\mathrm{CP}_{1}$ & $\mathrm{CP}_{2}$ & $\mathrm{CP}_{3}$ & $\mathrm{CP}_{4}$ & $\mathrm{CP}_{1}$ & $\mathrm{CP}_{2}$ & $\mathrm{CP}_{3}$ & $\mathrm{CP}_{4}$ \\
\hline $\mathrm{HQIC}_{1}$ & 10.2 & 18.2 & 4.3 & 3.9 & 20.9 & 33.0 & 15.5 & 5.6 & 28.0 & 49.5 & 35.0 & 7.7 \\
\hline $\mathrm{HQIC}_{2}$ & 8.3 & 19.8 & 6.9 & 8.5 & 16.6 & 34.3 & 26.0 & 16.8 & 23.6 & 40.0 & 39.9 & 18.4 \\
\hline $\mathrm{BIC}_{1}$ & 10.3 & 9.7 & 0.9 & 0.4 & 20.7 & 20.4 & 2.2 & 0.7 & 25.7 & 30.1 & 10.8 & 0.5 \\
\hline $\mathrm{BIC}_{2}$ & 16.2 & 16.4 & 3.7 & 2.6 & 26.2 & 28.3 & 10.8 & 2.1 & 32.2 & 47.2 & 20.4 & 5.8 \\
\hline $\mathrm{CAIC}_{1}$ & 9.6 & 6.9 & 0.2 & 0.1 & 17.3 & 15.9 & 1.4 & 0.4 & 27.3 & 31.8 & 5.3 & 0.1 \\
\hline $\mathrm{CAIC}_{2}$ & 13.4 & 11.3 & 1.5 & 0.9 & 24.6 & 24.8 & 4.9 & 1.9 & 31.6 & 44.5 & 12.2 & 1.9 \\
\hline \multicolumn{13}{|c|}{ REML estimation $(t=8)$} \\
\hline $\mathrm{AIC}_{1}$ & 14.9 & 37.0 & 40.3 & 89.2 & 19.1 & 45.0 & 63.3 & 98.6 & 20.5 & 45.9 & 67.5 & 99.8 \\
\hline $\mathrm{AIC}_{2}$ & 12.8 & 30.9 & 32.2 & 58.3 & 16.0 & 38.9 & 49.0 & 64.5 & 19.8 & 42.5 & 60.4 & 83.1 \\
\hline $\mathrm{AICc}_{1}$ & 19.4 & 52.0 & 50.7 & 84.5 & 21.5 & 52.2 & 73.4 & 98.3 & 20.2 & 48.8 & 73.3 & 99.9 \\
\hline $\mathrm{AICc}_{2}$ & 0.0 & 0.0 & 0.0 & 18.2 & 40.2 & 62.3 & 65.3 & 0.0 & 39.4 & 71.2 & 97.1 & 99.9 \\
\hline $\mathrm{AICc}_{3}$ & 17.7 & 42.4 & 38.6 & 51.3 & 18.5 & 41.0 & 56.9 & 62.5 & 19.2 & 44.8 & 65.2 & 82.2 \\
\hline $\mathrm{AICc}_{4}$ & 0.0 & 0.0 & 0.0 & 9.2 & 32.3 & 60.2 & 32.3 & 0.0 & 37.4 & 62.2 & 81.1 & 66.1 \\
\hline $\mathrm{HQIC}_{1}$ & 50.8 & 75.8 & 29.5 & 53.9 & 58.1 & 88.4 & 67.3 & 97.6 & 53.6 & 90.3 & 96.0 & 99.9 \\
\hline $\mathrm{HQIC}_{2}$ & 25.3 & 55.9 & 44.0 & 87.0 & 35.6 & 75.2 & 78.1 & 98.5 & 38.0 & 81.0 & 95.2 & 99.9 \\
\hline $\mathrm{HQIC}_{3}$ & 31.0 & 50.6 & 17.2 & 27.6 & 36.0 & 55.1 & 37.2 & 50.1 & 41.6 & 69.5 & 70.7 & 70.2 \\
\hline $\mathrm{HQIC}_{4}$ & 21.1 & 42.0 & 32.8 & 50.9 & 27.3 & 53.2 & 53.0 & 57.7 & 31.0 & 67.0 & 77.0 & 75.2 \\
\hline $\mathrm{BIC}_{1}$ & 82.9 & 71.7 & 2.0 & 1.0 & 84.0 & 97.5 & 25.6 & 29.5 & 87.4 & 99.2 & 74.8 & 94.4 \\
\hline $\mathrm{BIC}_{2}$ & 50.7 & 75.8 & 24.9 & 53.8 & 60.8 & 92.5 & 59.9 & 95.6 & 70.5 & 96.1 & 91.6 & 99.8 \\
\hline $\mathrm{BIC}_{3}$ & 31.8 & 33.3 & 0.9 & 0.6 & 36.0 & 38.4 & 8.8 & 11.2 & 47.3 & 53.2 & 38.9 & 51.0 \\
\hline $\mathrm{BIC}_{4}$ & 30.8 & 50.5 & 16.8 & 27.3 & 33.6 & 52.6 & 30.8 & 45.9 & 48.3 & 67.0 & 61.3 & 64.0 \\
\hline $\mathrm{CAIC}_{1}$ & 87.6 & 71.0 & 1.0 & 0.0 & 89.6 & 96.5 & 14.7 & 7.6 & 90.2 & 97.6 & 60.8 & 88.2 \\
\hline $\mathrm{CAIC}_{2}$ & 70.0 & 77.5 & 8.9 & 14.0 & 75.6 & 96.7 & 42.1 & 74.8 & 80.1 & 98.0 & 84.2 & 99.5 \\
\hline $\mathrm{CAIC}_{3}$ & 27.8 & 23.3 & 0.5 & 0.0 & 32.6 & 29.4 & 4.6 & 02.9 & 42.3 & 46.5 & 27.6 & 44.8 \\
\hline $\mathrm{CAIC}_{4}$ & 34.0 & 42.2 & 5.7 & 6.9 & 37.9 & 46.6 & 17.0 & 30.4 & 49.0 & 61.9 & 51.0 & 57.0 \\
\hline \multicolumn{13}{|c|}{ ML estimation $(t=8)$} \\
\hline AIC & 6.5 & 21.8 & 27.0 & 62.5 & 8.9 & 33.6 & 44.5 & 74.0 & 10.9 & 39.8 & 59.7 & 92.4 \\
\hline $\mathrm{AICc}_{1}$ & 0.0 & 0.0 & 0.0 & 0.0 & 21.5 & 52.1 & 31.0 & 0.0 & 26.4 & 60.5 & 81.1 & 77.6 \\
\hline $\mathrm{AICc}_{2}$ & 10.4 & 31.4 & 33.1 & 57.3 & 12.4 & 38.6 & 51.1 & 70.5 & 20.4 & 41.9 & 66.2 & 91.6 \\
\hline $\mathrm{HQIC}_{1}$ & 19.6 & 35.9 & 17.7 & 39.6 & 28.6 & 46.4 & 36.6 & 57.4 & 39.3 & 67.8 & 72.2 & 80.3 \\
\hline $\mathrm{HQIC}_{2}$ & 12.8 & 32.6 & 30.1 & 61.5 & 22.0 & 47.1 & 50.0 & 65.1 & 34.0 & 64.9 & 76.1 & 85.2 \\
\hline $\mathrm{BIC}_{1}$ & 20.6 & 23.8 & 1.5 & 1.9 & 27.8 & 31.9 & 11.3 & 15.2 & 43.9 & 51.5 & 36.7 & 61.2 \\
\hline $\mathrm{BIC}_{2}$ & 19.5 & 35.7 & 17.8 & 39.6 & 29.4 & 43.8 & 31.1 & 53.3 & 46.6 & 65.2 & 62.5 & 75.5 \\
\hline $\mathrm{CAIC}_{1}$ & 16.4 & 18.0 & 0.4 & 0.2 & 23.6 & 26.1 & 6.7 & 4.0 & 38.6 & 46.2 & 27.1 & 53.3 \\
\hline $\mathrm{CAIC}_{2}$ & 22.5 & 36.6 & 6.2 & 11.1 & 30.7 & 38.4 & 22.9 & 37.2 & 47.2 & 58.3 & 50.6 & 68.9 \\
\hline
\end{tabular}

See the note from Table 4

2) A comparison of the results for both normal and nonnormal distributions shows that the average differences were approximately 19 and 9 percentage points for the efficient and consistent criteria, respectively. As a consequence, when the data were obtained from a moderately skewed distribution the differences between the two classes of criteria with respect to selecting the correct model among a set of competing models were small.
3) Regardless of the total sample size, length of the repeated measures, and method of estimation used, the performance of the consistent criteria was better under simple covariance patterns than under complex covariance patterns, and vice versa for the efficient criteria.

4) In general, the consistent criteria based on $n$ were more effective when selecting the best model than the consistent criteria based on $m$, particularly for more complex covariance patterns. In contrast, the simula- 
Table 6 Percentages of correct identifications for normal data and reduced model $(t=4,8)$

\begin{tabular}{|c|c|c|c|c|c|c|c|c|c|c|c|c|}
\hline & \multicolumn{4}{|c|}{$n=30\left(n_{1}=n_{2}=15\right)$} & \multicolumn{4}{|c|}{$n=60\left(n_{1}=n_{2}=30\right)$} & \multicolumn{4}{|c|}{$n=120\left(n_{1}=n_{2}=60\right)$} \\
\hline & $\mathrm{CP}_{1}$ & $\mathrm{CP}_{2}$ & $\mathrm{CP}_{3}$ & $\mathrm{CP}_{4}$ & $\mathrm{CP}_{1}$ & $\mathrm{CP}_{2}$ & $\mathrm{CP}_{3}$ & $\mathrm{CP}_{4}$ & $\mathrm{CP}_{1}$ & $\mathrm{CP}_{2}$ & $\mathrm{CP}_{3}$ & $\mathrm{CP}_{4}$ \\
\hline \multicolumn{13}{|c|}{ REML estimation $(t=4)$} \\
\hline $\mathrm{AIC}_{1}$ & 0.0 & 0.0 & 0.0 & 0.0 & 0.5 & 0.0 & 0.0 & 0.0 & 0.0 & 0.0 & 0.0 & 0.0 \\
\hline $\mathrm{AIC}_{2}$ & 13.4 & 14.2 & 6.9 & 5.8 & 34.5 & 35.6 & 20.9 & 24.6 & 50.5 & 53.4 & 55.2 & 26.0 \\
\hline $\mathrm{AICc}_{1}$ & 0.0 & 0.0 & 0.0 & 0.0 & 0.0 & 0.0 & 0.0 & 0.0 & 0.0 & 0.0 & 0.0 & 0.0 \\
\hline $\mathrm{AICc}_{2} 0.0$ & 0.0 & 0.0 & 0.0 & 0.0 & 0.0 & 0.0 & 0.0 & 0.0 & 0.0 & 0.0 & 0.0 & \\
\hline $\mathrm{AICc}_{3}$ & 16.5 & 10.8 & 6.4 & 5.0 & 36.4 & 32.2 & 19.9 & 20.9 & 51.1 & 55.2 & 54.2 & 23.5 \\
\hline $\mathrm{AICc}_{4}$ & 52.4 & 25.4 & 1.7 & 0.2 & 58.9 & 40.8 & 15.7 & 19.7 & 63.2 & 66.7 & 54.2 & 20.1 \\
\hline $\mathrm{HQIC}_{1}$ & 0.0 & 0.0 & 0.0 & 0.0 & 0.0 & 0.0 & 0.0 & 0.0 & 0.0 & 0.0 & 0.0 & 0.0 \\
\hline $\mathrm{HQIC}_{2}$ & 0.0 & 0.0 & 0.0 & 0.0 & 0.0 & 0.0 & 0.0 & 0.0 & 0.0 & 0.0 & 0.0 & 0.0 \\
\hline $\mathrm{HQIC}_{3}$ & 52.3 & 24.8 & 4.2 & 2.5 & 69.6 & 39.0 & 8.2 & 10.3 & 81.3 & 73.9 & 45.1 & 8.3 \\
\hline $\mathrm{HQIC}_{4}$ & 34.6 & 22.5 & 6.5 & 7.6 & 65.9 & 42.2 & 17.7 & 15.8 & 79.9 & 73.3 & 48.3 & 10.9 \\
\hline $\mathrm{BIC}_{1}$ & 0.0 & 0.0 & 0.0 & 0.0 & 0.2 & 0.1 & 0.3 & 0.3 & 47.7 & 28.1 & 3.4 & 0.4 \\
\hline $\mathrm{BIC}_{2}$ & 0.0 & 0.0 & 0.0 & 0.0 & 0.0 & 0.0 & 0.0 & 0.0 & 0.0 & 0.0 & 0.0 & 0.0 \\
\hline $\mathrm{BIC}_{3}$ & 79.2 & 9.6 & 0.3 & 0.2 & 87.5 & 23.1 & 0.7 & 0.9 & 96.2 & 53.2 & 8.5 & 1.2 \\
\hline $\mathrm{BIC}_{4}$ & 68.8 & 24.1 & 1.7 & 1.7 & 86.8 & 34.9 & 3.6 & 5.9 & 92.4 & 68.4 & 20.9 & 3.5 \\
\hline $\mathrm{CAIC}_{1}$ & 12.0 & 0.0 & 0.0 & 0.0 & 58.1 & 8.1 & 0.0 & 0.2 & 74.5 & 33.3 & 1.5 & 0.3 \\
\hline $\mathrm{CAIC}_{2}$ & 0.0 & 0.0 & 0.0 & 0.0 & 0.0 & 0.0 & 0.0 & 0.0 & 17.6 & 15.8 & 3.5 & 0.2 \\
\hline $\mathrm{CAIC}_{3}$ & 84.9 & 5.9 & 0.2 & 0.1 & 92.8 & 17.4 & 0.0 & 0.4 & 97.8 & 42.2 & 3.7 & 0.7 \\
\hline $\mathrm{CAIC}_{4}$ & 83.7 & 15.5 & 0.6 & 0.8 & 91.5 & 26.6 & 1.2 & 2.5 & 95.9 & 57.0 & 10.4 & 1.6 \\
\hline \multicolumn{13}{|c|}{ ML estimation $(t=4)$} \\
\hline $\mathrm{AIC}$ & 47.0 & 26.7 & 16.7 & 13.8 & 51.6 & 43.3 & 30.2 & 20.7 & 55.9 & 57.9 & 53.7 & 31.6 \\
\hline $\mathrm{AICc}_{1}$ & 76.5 & 29.1 & 9.2 & 0.9 & 66.6 & 48.2 & 19.2 & 7.3 & 62.6 & 66.6 & 51.9 & 24.7 \\
\hline $\mathrm{AICc}_{2}$ & 54.4 & 27.2 & 15.8 & 10.0 & 55.4 & 44.9 & 29.7 & 18.3 & 58.1 & 60.1 & 53.8 & 30.2 \\
\hline $\mathrm{HQIC}_{1}$ & 74.0 & 20.1 & 7.9 & 4.6 & 82.2 & 42.7 & 14.9 & 7.6 & 85.7 & 74.8 & 33.3 & 10.9 \\
\hline $\mathrm{HQIC}_{2}$ & 59.6 & 25.3 & 14.4 & 8.8 & 73.3 & 45.6 & 22.3 & 10.5 & 79.8 & 73.7 & 41.5 & 16.3 \\
\hline $\mathrm{BIC}_{1}$ & 86.4 & 9.2 & 0.4 & 0.3 & 95.5 & 19.4 & 0.9 & 0.6 & 97.2 & 55.2 & 8.3 & 1.8 \\
\hline $\mathrm{BIC}_{2}$ & 78.8 & 18.6 & 2.7 & 1.1 & 88.0 & 34.5 & 7.3 & 2.2 & 92.7 & 69.7 & 20.9 & 9.1 \\
\hline $\mathrm{CAIC}_{1}$ & 91.8 & 3.8 & 0.1 & 0.1 & 97.2 & 12.1 & 0.5 & 0.2 & 98.0 & 45.0 & 4.4 & 0.4 \\
\hline $\mathrm{CAIC}_{2}$ & 86.6 & 11.1 & 1.3 & 0.7 & 94.2 & 23.6 & 3.3 & 1.4 & 96.5 & 60.5 & 12.7 & 2.3 \\
\hline \multicolumn{13}{|c|}{ REML estimation $(t=8)$} \\
\hline $\mathrm{AIC}_{1}$ & 0.0 & 0.8 & 0.0 & 5.2 & 0.0 & 0.0 & 0.0 & 0.4 & 0.0 & 0.0 & 0.0 & 0.3 \\
\hline $\mathrm{AIC}_{2}$ & 57.9 & 36.3 & 42.4 & 71.5 & 93.5 & 57.4 & 73.7 & 88.9 & 97.3 & 70.7 & 84.2 & 92.4 \\
\hline $\mathrm{AICc}_{1}$ & 0.0 & 0.6 & 0.0 & 0.0 & 0.0 & 0.0 & 0.0 & 0.0 & 0.0 & 0.0 & 0.0 & 0.0 \\
\hline $\mathrm{AICc}_{2}$ & 0.0 & 0.5 & 0.0 & 0.8 & 0.0 & 0.0 & 0.0 & 0.0 & 0.0 & 0.0 & 0.0 & 0.0 \\
\hline $\mathrm{AICc}_{3}$ & 36.6 & 45.5 & 42.9 & 73.3 & 51.0 & 62.9 & 74.4 & 86.5 & 61.4 & 71.0 & 84.7 & 92.8 \\
\hline $\mathrm{AICc}_{4}$ & 0.2 & 0.2 & 0.0 & 88.7 & 59.4 & 87.0 & 82.0 & 0.0 & 65.7 & 83.8 & 96.0 & 95.0 \\
\hline $\mathrm{HQIC}_{1}$ & 0.0 & 0.3 & 0.0 & 0.6 & 0.0 & 0.0 & 0.0 & 0.0 & 0.0 & 0.0 & 0.0 & 0.0 \\
\hline $\mathrm{HQIC}_{2}$ & 0.0 & 0.4 & 0.0 & 0.2 & 0.0 & 0.0 & 0.0 & 0.0 & 0.0 & 0.0 & 0.0 & 0.0 \\
\hline $\mathrm{HQIC}_{3}$ & 76.5 & 76.8 & 27.0 & 43.6 & 81.5 & 88.8 & 71.0 & 94.1 & 88.4 & 93.2 & 94. & 96.6 \\
\hline $\mathrm{HQIC}_{4}$ & 53.0 & 58.7 & 43.7 & 75.0 & 70.6 & 80.3 & 78.8 & 92.8 & 82.0 & 88.9 & 92.0 & 95.7 \\
\hline $\mathrm{BIC}_{1}$ & 0.0 & 0.9 & 0.0 & 1.9 & 0.2 & 0.0 & 0.0 & 16.0 & 0.0 & 0.0 & 1.8 & 70.6 \\
\hline $\mathrm{BIC}_{2}$ & 0.0 & 0.6 & 0.0 & 3.8 & 0.2 & 0.0 & 0.0 & 0.6 & 0.0 & 0.0 & 0.0 & 0.2 \\
\hline $\mathrm{BIC}_{3}$ & 89.4 & 74.4 & 1.8 & 7.0 & 94.1 & 97.6 & 24.5 & 29.4 & 98.0 & 99.3 & 84.8 & 99.0 \\
\hline $\mathrm{BIC}_{4}$ & 76.8 & 76.5 & 26.8 & 43.6 & 85.9 & 91.9 & 63.6 & 93.3 & 93.6 & 97.0 & 94.6 & 98.5 \\
\hline $\mathrm{CAIC}_{1}$ & 0.0 & 0.4 & 0.0 & 1.6 & 1.8 & 3.6 & 4.6 & 8.7 & 50.8 & 54.4 & 48.3 & 83.2 \\
\hline
\end{tabular}


Table 6 (continued)

\begin{tabular}{|c|c|c|c|c|c|c|c|c|c|c|c|c|}
\hline & \multicolumn{4}{|c|}{$n=30\left(n_{1}=n_{2}=15\right)$} & \multicolumn{4}{|c|}{$n=60\left(n_{1}=n_{2}=30\right)$} & \multicolumn{4}{|c|}{$n=120\left(n_{1}=n_{2}=60\right)$} \\
\hline & $\mathrm{CP}_{1}$ & $\mathrm{CP}_{2}$ & $\mathrm{CP}_{3}$ & $\mathrm{CP}_{4}$ & $\mathrm{CP}_{1}$ & $\mathrm{CP}_{2}$ & $\mathrm{CP}_{3}$ & $\mathrm{CP}_{4}$ & $\mathrm{CP}_{1}$ & $\mathrm{CP}_{2}$ & $\mathrm{CP}_{3}$ & $\mathrm{CP}_{4}$ \\
\hline $\mathrm{CAIC}_{2}$ & 0.0 & 0.0 & 0.0 & 2.8 & 0.2 & 0.0 & 0.0 & 5.3 & 0.0 & 0.0 & 0.0 & 29.9 \\
\hline $\mathrm{CAIC}_{3}$ & 90.1 & 59.4 & 0.2 & 3.7 & 95.7 & 98.7 & 11.2 & 5.9 & 98.9 & 99.5 & 74.1 & 98.4 \\
\hline $\mathrm{CAIC}_{4}$ & 85.8 & 79.1 & 8.5 & 9.0 & 90.6 & 95.0 & 44.9 & 74.2 & 96.4 & 98.6 & 92.8 & 99.0 \\
\hline \multicolumn{13}{|c|}{ ML estimation $(t=8)$} \\
\hline AIC & 56.7 & 60.8 & 57.6 & 73.6 & 59.7 & 67.6 & 79.9 & 79.3 & 62.0 & 70.7 & 82.3 & 81.5 \\
\hline $\mathrm{AICc}_{1}$ & 0.1 & 0.0 & 0.0 & 79.2 & 64.3 & 83.4 & 75.6 & 0.4 & 69.8 & 88.6 & 89.2 & 95.4 \\
\hline $\mathrm{AICc}_{2}$ & 61.6 & 69.4 & 58.5 & 74.9 & 62.1 & 71.5 & 81.5 & 82.6 & 63.4 & 73.0 & 83.5 & 83.3 \\
\hline $\mathrm{HQIC}_{1}$ & 84.9 & 86.5 & 27.0 & 49.0 & 87.8 & 92.7 & 74.4 & 90.0 & 89.1 & 92.4 & 93.6 & 93.5 \\
\hline $\mathrm{HQIC}_{2}$ & 70.6 & 73.2 & 53.7 & 73.9 & 79.0 & 85.8 & 82.2 & 85.5 & 82.8 & 88.8 & 91.2 & 90.7 \\
\hline $\mathrm{BIC}_{1}$ & 93.3 & 80.5 & 1.8 & 2.3 & 96.5 & 98.2 & 26.1 & 33.6 & 97.9 & 98.9 & 85.0 & 99.2 \\
\hline $\mathrm{BIC}_{2}$ & 84.6 & 86.6 & 27.6 & 49.0 & 89.8 & 94.4 & 67.0 & 90.2 & 93.2 & 96.2 & 94.9 & 96.4 \\
\hline $\mathrm{CAIC}_{1}$ & 92.2 & 66.1 & 0.1 & 1.2 & 98.2 & 98.6 & 12.2 & 7.3 & 98.6 & 99.5 & 74.0 & 98.8 \\
\hline $\mathrm{CAIC}_{2}$ & 89.8 & 87.1 & 9.3 & 12.2 & 93.8 & 97.0 & 46.6 & 77.6 & 95.8 & 98.0 & 92.6 & 97.9 \\
\hline
\end{tabular}

See the note from Table 4

Table 7 Percentages of correct identifications for non-normal data and reduced model $(t=4,8)$

\begin{tabular}{|c|c|c|c|c|c|c|c|c|c|c|c|c|}
\hline & \multicolumn{4}{|c|}{$n=30\left(n_{1}=n_{2}=15\right)$} & \multicolumn{4}{|c|}{$n=60\left(n_{1}=n_{2}=30\right)$} & \multicolumn{4}{|c|}{$n=120\left(n_{1}=n_{2}=60\right)$} \\
\hline & $\mathrm{CP}_{1}$ & $\mathrm{CP}_{2}$ & $\mathrm{CP}_{3}$ & $\mathrm{CP}_{4}$ & $\mathrm{CP}_{1}$ & $\mathrm{CP}_{2}$ & $\mathrm{CP}_{3}$ & $\mathrm{CP}_{4}$ & $\mathrm{CP}_{1}$ & $\mathrm{CP}_{2}$ & $\mathrm{CP}_{3}$ & $\mathrm{CP}_{4}$ \\
\hline \multicolumn{13}{|c|}{ REML estimation $(t=4)$} \\
\hline $\mathrm{AIC}_{1}$ & 0.0 & 0.0 & 0.0 & 0.0 & 0.0 & 0.0 & 0.0 & 0.0 & 0.0 & 0.0 & 0.0 & 0.0 \\
\hline $\mathrm{AIC}_{2}$ & 2.2 & 8.5 & 4.5 & 5.2 & 10.7 & 20.5 & 20.3 & 19.9 & 12.5 & 29.9 & 37.5 & 23.4 \\
\hline $\mathrm{AICc}_{1}$ & 0.0 & 0.0 & 0.0 & 0.0 & 0.0 & 0.0 & 0.0 & 0.0 & 0.0 & 0.0 & 0.0 & 0.0 \\
\hline $\mathrm{AICc}_{2}$ & 0.0 & 0.0 & 0.0 & 0.0 & 0.0 & 0.8 & 0.0 & 0.0 & 0.0 & 0.0 & 0.0 & 0.0 \\
\hline $\mathrm{AICc}_{3}$ & 4.7 & 8.5 & 5.9 & 5.1 & 11.6 & 22.0 & 21.2 & 17.2 & 13.1 & 30.1 & 38.7 & 22.5 \\
\hline $\mathrm{AICc}_{4}$ & 17.8 & 30.9 & 3.0 & 0.3 & 30.3 & 37.8 & 21.1 & 15.5 & 37.2 & 38.8 & 44.0 & 22.3 \\
\hline $\mathrm{HQIC}_{1}$ & 0.0 & 0.0 & 0.0 & 0.0 & 0.0 & 0.0 & 0.0 & 0.0 & 0.0 & 0.0 & 0.0 & 0.0 \\
\hline $\mathrm{HQIC}_{2}$ & 0.0 & 0.0 & 0.0 & 0.0 & 0.0 & 0.0 & 0.0 & 0.0 & 0.0 & 0.0 & 0.0 & 0.0 \\
\hline $\mathrm{HQIC}_{3}$ & 36.2 & 23.7 & 5.6 & 4.3 & 42.0 & 41.6 & 16.3 & 6.2 & 43.3 & 60.6 & 43.6 & 11.9 \\
\hline $\mathrm{HQIC}_{4}$ & 19.3 & 14.2 & 7.1 & 6.4 & 30.9 & 36.2 & 23.5 & 9.8 & 37.9 & 52.7 & 46.2 & 16.0 \\
\hline $\mathrm{BIC}_{1}$ & 0.0 & 0.0 & 0.0 & 0.0 & 0.7 & 1.2 & 0.7 & 0.1 & 25.7 & 26.1 & 11.4 & 1.1 \\
\hline $\mathrm{BIC}_{2}$ & 0.0 & 0.0 & 0.0 & 0.0 & 0.0 & 0.0 & 0.0 & 0.0 & 0.0 & 0.0 & 0.0 & 0.0 \\
\hline $\mathrm{BIC}_{3}$ & 60.5 & 25.1 & 2.1 & 0.7 & 71.5 & 44.1 & 5.3 & 1.3 & 77.2 & 70.9 & 20.6 & 2.5 \\
\hline $\mathrm{BIC}_{4}$ & 47.9 & 25.8 & 5.3 & 3.2 & 59.2 & 45.3 & 11.8 & 4.6 & 64.4 & 68.7 & 36.5 & 5.9 \\
\hline $\mathrm{CAIC}_{1}$ & 8.3 & 4.7 & 0.3 & 0.0 & 43.0 & 22.8 & 2.2 & 0.5 & 58.5 & 47.4 & 11.1 & 0.8 \\
\hline $\mathrm{CAIC}_{2}$ & 0.0 & 0.0 & 0.0 & 0.0 & 0.0 & 0.1 & 0.3 & 0.0 & 3.2 & 8.3 & 8.5 & 0.5 \\
\hline $\mathrm{CAIC}_{3}$ & 71.3 & 21.9 & 0.8 & 0.1 & 83.3 & 40.7 & 3.9 & 0.8 & 83.8 & 65.8 & 14.2 & 1.1 \\
\hline $\mathrm{CAIC}_{4}$ & 58.5 & 25.9 & 2.7 & 1.1 & 74.9 & 45.6 & 7.8 & 1.9 & 78.9 & 71.5 & 24.1 & 3.6 \\
\hline \multicolumn{13}{|c|}{ ML estimation $(t=4)$} \\
\hline AIC & 17.2 & 21.3 & 11.3 & 19.3 & 17.0 & 35.4 & 25.4 & 22.6 & 20.3 & 34.9 & 39.8 & 29.1 \\
\hline $\mathrm{AICc}_{1}$ & 44.1 & 34.2 & 3.4 & 0.4 & 27.8 & 50.8 & 22.2 & 10.2 & 29.0 & 40.6 & 46.6 & 23.0 \\
\hline $\mathrm{AICc}_{2}$ & 21.6 & 27.5 & 10.5 & 13.5 & 19.5 & 38.0 & 25.7 & 20.3 & 23.0 & 35.1 & 42.3 & 27.3 \\
\hline
\end{tabular}


Table 7 (continued)

\begin{tabular}{|c|c|c|c|c|c|c|c|c|c|c|c|c|}
\hline & \multicolumn{4}{|c|}{$n=30\left(n_{1}=n_{2}=15\right)$} & \multicolumn{4}{|c|}{$n=60\left(n_{1}=n_{2}=30\right)$} & \multicolumn{4}{|c|}{$n=120\left(n_{1}=n_{2}=60\right)$} \\
\hline & $\mathrm{CP}_{1}$ & $\mathrm{CP}_{2}$ & $\mathrm{CP}_{3}$ & $\mathrm{CP}_{4}$ & $\mathrm{CP}_{1}$ & $\mathrm{CP}_{2}$ & $\mathrm{CP}_{3}$ & $\mathrm{CP}_{4}$ & $\mathrm{CP}_{1}$ & $\mathrm{CP}_{2}$ & $\mathrm{CP}_{3}$ & $\mathrm{CP}_{4}$ \\
\hline $\mathrm{HQIC}_{1}$ & 39.0 & 30.8 & 6.7 & 5.7 & 43.9 & 53.2 & 16.4 & 8.1 & 48.8 & 64.8 & 46.0 & 11.5 \\
\hline $\mathrm{HQIC}_{2}$ & 25.2 & 27.6 & 10.0 & 13.0 & 32.2 & 49.6 & 21.1 & 12.8 & 41.5 & 56.7 & 49.4 & 16.7 \\
\hline $\mathrm{BIC}_{1}$ & 63.6 & 25.0 & 2.5 & 0.5 & 72.0 & 49.4 & 4.9 & 1.5 & 78.0 & 70.2 & 22.1 & 2.9 \\
\hline $\mathrm{BIC}_{2}$ & 44.5 & 30.6 & 5.5 & 3.5 & 53.7 & 52.5 & 11.5 & 4.7 & 67.8 & 71.7 & 35.5 & 6.5 \\
\hline $\mathrm{CAIC}_{1}$ & 72.2 & 23.4 & 1.1 & 0.1 & 80.6 & 44.1 & 2.3 & 0.6 & 84.3 & 68.0 & 13.8 & 1.2 \\
\hline $\mathrm{CAIC}_{2}$ & 59.8 & 26.5 & 3.2 & 1.6 & 68.9 & 51.6 & 6.2 & 2.9 & 76.5 & 71.5 & 24.9 & 3.7 \\
\hline \multicolumn{13}{|c|}{ REML estimation $(t=8)$} \\
\hline $\mathrm{AIC}_{1}$ & 0.0 & 0.0 & 0.0 & 23.8 & 0.0 & 0.0 & 0.0 & 2.3 & 0.0 & 0.0 & 0.0 & 4.0 \\
\hline $\mathrm{AIC}_{2}$ & 6.7 & 21.1 & 27.0 & 68.4 & 33.8 & 30.4 & 47.0 & 81.6 & 36.3 & 35.8 & 58.6 & 90.5 \\
\hline $\mathrm{AICc}_{1}$ & 0.0 & 0.0 & 0.0 & 32.4 & 0.0 & 0.0 & 0.0 & 12.3 & 0.0 & 0.0 & 0.0 & 4.0 \\
\hline $\mathrm{AICc}_{2}$ & 0.0 & 0.0 & 0.0 & 36.1 & 0.0 & 0.0 & 0.0 & 6.7 & 0.0 & 0.0 & 0.0 & 3.9 \\
\hline $\mathrm{AICc}_{3}$ & 7.8 & 30.6 & 36.5 & 67.4 & 11.4 & 40.1 & 54.6 & 82.6 & 19.2 & 44.2 & 62.8 & 90.8 \\
\hline $\mathrm{AICc}_{4}$ & 0.2 & 0.8 & 0.2 & 75.0 & 20.3 & 77.2 & 75.4 & 16.5 & 23.8 & 71.6 & 91.6 & 93.0 \\
\hline $\mathrm{HQIC}_{1}$ & 0.0 & 0.0 & 0.0 & 19.6 & 0.0 & 0.0 & 0.0 & 11.7 & 0.0 & 0.0 & 0.0 & 3.7 \\
\hline $\mathrm{HQIC}_{2}$ & 0.0 & 0.0 & 0.0 & 22.4 & 0.0 & 0.0 & 0.0 & 11.9 & 0.0 & 0.0 & 0.0 & 3.8 \\
\hline $\mathrm{HQIC}_{3}$ & 39.6 & 62.9 & 30.3 & 53.8 & 46.7 & 78.5 & 64.5 & 86.6 & 49.0 & 75.5 & 90.1 & 95.0 \\
\hline $\mathrm{HQIC}_{4}$ & 16.9 & 37.0 & 39.4 & 68.1 & 25.9 & 60.8 & 68.0 & 84.6 & 35.1 & 74.0 & 87.0 & 93.8 \\
\hline $\mathrm{BIC}_{1}$ & 0.0 & 0.0 & 0.0 & 22.5 & 0.0 & 0.0 & 0.0 & 25.7 & 0.0 & 0.0 & 0.9 & 67.4 \\
\hline $\mathrm{BIC}_{2}$ & 0.0 & 0.0 & 0.0 & 19.7 & 0.0 & 0.0 & 0.0 & 11.6 & 0.0 & 0.0 & 0.0 & 3.9 \\
\hline $\mathrm{BIC}_{3}$ & 70.6 & 75.8 & 7.9 & 12.0 & 80.5 & 95.7 & 29.3 & 51.8 & 86.4 & 98.1 & 76.0 & 96.5 \\
\hline $\mathrm{BIC}_{4}$ & 39.8 & 63.1 & 31.6 & 54.1 & 56.5 & 84.4 & 60.0 & 86.0 & 65.6 & 92.3 & 89.6 & 96.0 \\
\hline $\mathrm{CAIC}_{1}$ & 0.7 & 0.4 & 0.1 & 9.3 & 0.8 & 5.8 & 4.2 & 25.1 & 37.9 & 47.8 & 38.0 & 79.9 \\
\hline $\mathrm{CAIC}_{2}$ & 0.0 & 0.0 & 0.0 & 14.8 & 0.0 & 0.0 & 0.0 & 12.8 & 0.0 & 0.0 & 0.0 & 23.0 \\
\hline $\mathrm{CAIC}_{3}$ & 78.7 & 73.3 & 4.7 & 9.4 & 87.0 & 96.6 & 16.7 & 37.6 & 91.6 & 99.0 & 65.6 & 85.0 \\
\hline $\mathrm{CAIC}_{4}$ & 61.0 & 74.6 & 22.4 & 24.6 & 70.6 & 92.9 & 45.8 & 73.0 & 77.5 & 93.7 & 85.3 & 96.4 \\
\hline \multicolumn{13}{|c|}{ ML estimation $(t=8)$} \\
\hline $\mathrm{AIC}$ & 10.7 & 30.9 & 31.5 & 65.2 & 8.7 & 35.5 & 51.4 & 73.9 & 12.4 & 36.9 & 54.8 & 79.1 \\
\hline $\mathrm{AICc}_{1}$ & 0.1 & 1.1 & 0.0 & 86.3 & 27.2 & 61.2 & 69.0 & 27.0 & 38.6 & 83.6 & 87.3 & 94.0 \\
\hline $\mathrm{AICc}_{2}$ & 16.6 & 40.4 & 43.9 & 68.4 & 10.5 & 41.3 & 62.2 & 79.6 & 12.5 & 44.2 & 60.2 & 81.2 \\
\hline $\mathrm{HQIC}_{1}$ & 44.8 & 71.7 & 31.4 & 57.7 & 46.6 & 83.6 & 68.7 & 85.2 & 52.4 & 86.2 & 90.8 & 91.4 \\
\hline $\mathrm{HQIC}_{2}$ & 22.6 & 47.5 & 39.0 & 67.1 & 29.8 & 63.7 & 71.8 & 81.0 & 33.9 & 74.0 & 87.0 & 88.9 \\
\hline $\mathrm{BIC}_{1}$ & 81.3 & 80.8 & 6.6 & 11.5 & 83.4 & 96.4 & 32.2 & 46.6 & 87.6 & 98.2 & 79.1 & 96.4 \\
\hline $\mathrm{BIC}_{2}$ & 44.6 & 72.9 & 31.4 & 57.2 & 54.2 & 89.1 & 62.9 & 85.8 & 66.1 & 93.7 & 89.8 & 94.3 \\
\hline $\mathrm{CAIC}_{1}$ & 87.2 & 75.0 & 2.7 & 7.8 & 91.3 & 97.4 & 18.5 & 29.3 & 91.3 & 99.0 & 61.2 & 83.5 \\
\hline $\mathrm{CAIC}_{2}$ & 65.9 & 80.6 & 18.5 & 31.3 & 72.1 & 95.0 & 49.0 & 77.1 & 80.2 & 96.8 & 85.4 & 95.6 \\
\hline
\end{tabular}

See the note from Table 4

tion results pointed towards the use of $m$ in the penalty terms of the AICc when the true covariance structures were TOEPH and UN and towards the use of $n$ when the true covariance structures were AR and $\mathrm{ARH}$, although overall differences were generally small in this case.

5) Finally, the five information criteria and their examined variations performed slightly better under REML than under ML estimation. The average differences between the two methods of estimation were approximately
8 percentage points for the efficient criteria and approximately 15 percentage points for the consistent criteria. It is also noteworthy that the ability of criteria to select the correct model was superior under REML ${ }_{1}$ than under REML 2 . The average differences between the two versions of REML estimation were approximately 8 percentage points for the efficient criteria and approximately 18 percentage points for the consistent criteria. 


\section{Performances associated with normal data and reduced model}

The percentages of correct decisions when the data were normally distributed and the reduced model was adopted are found in Table 6 . These results can be summarized as follows:

1) Contrary to what happened when the data were generated from a full model, it is very apparent that in this case $\mathrm{REML}_{1}$-based information criteria are not appropriate for selecting the best model. A comparison of the results for both REML methods shows that the average differences were approximately 46 and 48 percentage points for the efficient and consistent criteria, respectively

2) The overall performance of the criteria under $R E M L_{2}$ estimation was roughly equivalent to the performance of the criteria under ML estimation. Specifically, the success rates averaged across covariance structures, number of subjects, and length of the repeated measures were 51.4 and $53.4 \%$ for the AIC, 46.8 and $50.7 \%$ for the AICc, 52.5 and $53.9 \%$ for the BIC, 48.9 and $49.8 \%$ for the CAIC, and 57.1 and $59.4 \%$ for the HQIC under $\mathrm{REML}_{2}$ and ML, respectively. Results also show that the performance of the consistent criteria was better under simple covariance patterns than under complex covariance patterns, and vice versa for the efficient criteria.

3) Finally, results also show that the consistent criteria based on $n$ performed better than the consistent criteria based on $m$ when the true covariance structures were TOEPH and UN, and vice versa when the true covariance structures were AR and ARH. On the other hand, the simulation results pointed towards the use of $m$ in the penalty terms of the AICc when the true covariance structures were TOEPH and UN and towards the use of $n$ when the true covariance structures were AR and ARH.

\section{Performances associated with non-normal data and reduced model}

The percentages of correct decisions when the data were obtained from a moderately skewed distribution and the reduced model was adopted are found in Table 7 . These results can be summarized as follows:

1) In general, the results obtained for the non-normal distributions were qualitatively similar to those found for the normal distributions. However, from a quantitative point of view the shape of the distribution substantially altered the performance of the efficient criteria and only slightly altered the performance of the consistent criteria, regardless of whether ML or REML estimation was used.

2) A comparison of the results for both normal and nonnormal distributions shows that the average differences were approximately 17 and six percentage points for the efficient and consistent criteria, respectively. Thus, the consistent criteria performed better overall than their efficient counterparts. In fact, a careful examination of Table 7 shows that the success rates averaged across covariance structures, number of subjects, length of the repeated measures, and estimation methods (ML and $\mathrm{REML}_{2}$ ), were $31.6 \%$ for the AIC, $34.8 \%$ for the $\mathrm{AICc}, 49.9 \%$ for the BIC, $49.3 \%$ for the CAIC, and $45.4 \%$ for the HQIC.

3) The performance of the five criteria was substantially better under REML $L_{2}$ than under $\mathrm{REML}_{1}$ estimation. As occurred for the reduced model and normally distributed data, the REML $L_{1}$ function was not supported here. Nevertheless, it is very apparent that the pattern of correct identifications obtained under $\mathrm{REML}_{2}$ closely resembled that obtained under ML. Specifically, the success rates averaged across covariance structures, total sample size, and length of the repeated measures were 30.8 and $31.6 \%$ for the AIC, 33.2 and $34.8 \%$ for the AICc, 49.2 and $49.9 \%$ for the BIC, 48.9 and $49.3 \%$ for the CAIC, and 44.8 and $45.4 \%$ for the HQIC under $\mathrm{REML}_{2}$ and ML, respectively.

4) Finally, under this condition, it is also noteworthy that the consistent criteria based on $n$ performed better than the consistent criteria based on $m$ when the true covariance structures were TOEPH and $\mathrm{UN}$, and vice versa when the true covariance structures were $A R$ and ARH. In contrast, our results pointed towards the use of $m$ in the penalty terms of the AICc when the true covariance structures were TOEPH and UN and towards the use of $n$ when the true covariance structures were AR and ARH.

\section{Discussion and recommendations}

The purpose of this study was to examine the relative merits of several information criteria in selecting the best regression model when both the mean structure and the covariance structure are unknown. Unbalanced designs due to missing data involving both a moderate and large number of repeated measurements and varying total sample sizes were investigated. Also, the study investigated the impact of using different methods of estimation for information criteria, the impact of different adjustments for calculating the information criteria, and the impact of different distribution shapes. Results of this investigation are consistent with those obtained in past studies. More- 
over, they contribute new findings that may aid researchers in the model selection process.

Simulation results showed that none of the procedures examined behaved correctly under all the conditions. Fortunately, despite differences in performance among the five criteria and their examined variations, performance of all criteria substantially improved with increased sample size and length of repeated measures. Averaging across the covariance structures, type of models, methods of estimation, length of repeated measures, and distribution shape the overall success rates for sample size configurations of $\left(n_{l}\right.$, $\left.n_{2}\right)=(15,15),\left(n_{1}, n_{2}\right)=(30,30)$, and $\left(n_{1}, n_{2}\right)=(60,60)$ were as follows: AIC, 29, 40, and 48\%; AICc, 21, 36, and $52 \%$; BIC, 25,36 , and $52 \%$; CAIC, 22,32 , and $51 \%$; and HQIC, 30, 42, and 55\%. Although no single criterion can be uniformly recommended, the AIC and HQIC had the best overall performance among the procedures examined. HQIC should in fact perform better in this study because selection is from a finite set of models (Burnham \& Anderson, 2002).

From a quantitative standpoint, this pattern of results indicates that the percentages of times that the model generating the data was chosen by the information criteria were lower than those obtained in Gurka's (2006) similar study. However, Gurka (2006), in addition to considering only a normal and large complete-data sample size case, allowed a choice between only six candidate models, and in our study 36 candidate models were fit for each generated dataset. Consequently, poor performance for selecting the true model is not surprising given that several of the models used in this study may provide good approximations.

On the other hand, from a qualitative standpoint, our results partially corroborated findings by Gurka (2006), who reported that the criteria performed better or equally well under REML estimation compared to ML estimation when choosing the proper mean and covariance structure simultaneously. In fact, Gurka (2006) suggested using $\mathrm{REML}_{1}$ estimation for the consistent criteria in selecting the proper model, and using $\mathrm{REML}_{2}$ estimation for the efficient criteria. Note, however, that our results indicated that while $\mathrm{REML}_{2}$-based information criteria performed comparably to the ML-based information criteria, performance of criteria was worse under $\mathrm{REML}_{1}$ than ML. Thus, Gurka's recommendation was not unequivocally supported here.

A possible explanation for this discrepancy is that in Gurka's study datasets were simulated using the uniform correlation structure commonly assumed in designs where the within-subject factor is randomly allocated to subjects. According to this model, variances are constant across time as are the correlations between any pair of measurements. This assumption is insufficient and often unrealistic for describing real-time series data in the health and behavioral sciences (Fitzmaurice et al., 2004; Molenberghs \& Kenward, 2007).

Beyond this, we also found that overall the consistent criteria (BIC, CAIC, and HQIC) performed better than their efficient counterparts (AIC and AICC) when the covariance patterns used to generate the data were relatively simple (i.e., AR and ARH). In contrast, the efficient criteria performed better than their consistent counterparts when the covariance patterns used to generate the data were more complex (i.e., TOEPH and UN). The consistent criteria tended to select a simpler model than the true model, particularly for the BIC and CAIC when the number of repeated measures was smaller. The opposite was true for the efficient criteria. Because excessively parsimonious structures are rarely adequate in real problems, selecting a model with few parameters is a type of error more severe than selecting a model with too many parameters. Hence, at least for the $\mathrm{AIC}$ and BIC, this result is consistent with the findings of Keselman, Algina et al. (1998) and Gomez et al. (2005).

With regard to discrepancies in the formulas involving in the penalty term of the criteria, our simulation studies showed that, regardless of whether ML or REML estimation had been used, the consistent criteria based on total number of subjects $(n)$ were more effective when selecting the best model than the consistent criteria based on total number of observations $(m)$, particularly for the BIC and CAIC criteria. These results corroborate and generalize those found in Gurka (2006) study, while confirming the recommendation to use $n$ in the penalty term of the BIC and CAIC criteria (see, Carlin \& Louis, 2001; Kass \& Raftery, 1995). In contrast, the simulation results pointed towards the use of $m$ in the penalty terms of the AICc, as used by Proc Mixed in SAS and mixed command in SPSS/PASW. As indicated above, sample size in SAS is equal to $n$ whereas sample size in SPSS/PASW is equal to $m$ under ML and REML, respectively, when computing the BIC and CAIC.

The simulation studies covered in this paper also revealed that the criteria exhibited a clear superiority in their ability to accurately select the correct model when data were obtained from normal distributions. However, none of the procedures considered performed well when data were obtained from moderately skewed distributions, regardless of whether ML or REML estimation was used. For this scenario, consistent criteria and, even more so, efficient criteria, picked the wrong model more frequently than the correct model. This finding serves to reinforce the importance of testing for evidence of skewness in the data (see Vallejo, Ato, \& Fernández, 2010, for details).

To conclude, we would like to add four brief comments. First and foremost, it is very important to emphasize that the error rate for all criteria decreased as the sample size 
increased. Therefore, researchers interested in carrying out studies that have sufficient power to detect the model closest to the true data generating process, should avoid using small sample sizes whenever possible. In order to reach an acceptable power to distinguish between competing models, the rule of thumb $n \geq 10 t$ per group is suggested. Second, with small samples the standard selection criteria may be highly inefficient, particularly if some data are missing and/or the form of the matrix plays an important role in the estimation. In these cases, it may be more appropriate to select the best mean model assuming the unstructured pattern to model the within-subject errors in the analysis (Siddiqui, Hung, \& O'Neill, 2009). Third, it should be noted that information criteria do not automatically select the best model from all possible candidate models. Thus, the joint modeling of mean and covariance structures can be impractical if the number of explanatory variables is large. Fourth, the results are of course limited to the conditions examined in our study, though we sense that they may be generalizable to a considerably wider range of conditions that could conceivably be obtained in behavioral science research. We conclude by noting that this study did not address the effects of heterogeneity of covariance across groups on the performance of the criteria in selecting the best regression model. Based on this research, it can be expected that between-groups heterogeneity will substantially affect the consistent criteria's performance. In future research, it would be informative to examine the performance of the linear model, using techniques (e.g., generalized linear mixed models) that allow for nonnormal error term distributions and relax the requirement of constant variability. Currently, SAS Proc Glimmix allows users to fit statistical models to data when assumptions of normality and variance homogeneity are not necessarily satisfied.

Author Note This work was supported by a Grant PSI2008-03624 from the Spanish Ministry of Science and Innovation. We gratefully thank the anonymous reviewers and the associate editor for their helpful comments and very constructive suggestions.

\section{References}

Akaike, H. (1974). A new look at the statistical model identification. IEEE Transaction on Automatic Control, AC-19, 716-723. doi:10.1109/TAC.1974.1100705

Algina, J., \& Keselman, H. J. (2004). A comparison of methods for longitudinal analysis with missing data. Journal of Modern Applied Statistical Methods, 3, 13-26.

Azari, R., Li, L., \& Tsai, C. L. (2006). Longitudinal data model selection. Computational Statistics and Data Analysis, 50, 30533066. doi:10.1016/j.csda.2005.05.009

Bozdogan, H. (1987). Model selection and Akaike's Information Criterion (AIC): The general theory and its analytical extensions. Psychometrika, 52, 345-370. doi:10.1007/BF02294361
Bozdogan, H. (2000). Akaike's information criterion and recent developments information complexity. Journal of Mathematical Psychology, 44, 62-91. doi:10.1006/jmps.1999.1277

Burnham, K. P., \& Anderson, D. R. (2002). Model selection and multimodel inference. A practical information-theoretic approach. New York, NY: Springer.

Carlin, B. P., \& Louis, T. A. (2001). Bayes and empirical Bayes methods for data analysis (2nd ed.). London: Chapman \& Hall / CRC Press.

Claeskens, G., \& Hjort, N. L. (2008). Model selection and model averaging. New York, NY: Cambridge University Press.

Cooper, D. M. K., \& Thompson, R. (1977). A note on the estimation of the parameters of the autoregressive-moving average process. Biometrika, 64, 625-628. doi:10.1093/biomet/64.3.625

DeSouza, C. M., Legedza, A. T., \& Sankoh, A. J. (2009). An overview of practical approaches for handling missing data in clinical trials. Journal of Biopharmaceutical Statistics, 6, 10551073. doi:10.1080/10543400903242795

Feng, R., Zhou, G., Zhang, M., \& Zhang, H. (2009). Analysis of twin data using SAS. Biometrics, 65, 584-589. doi:10.1111/j.15410420.2008.01098.x

Ferron, J., Dailey, R., \& Yi, Q. (2002). Effects of misspecifying the first-level error structure in two-level models of change. Multivariate Behavioral Research, 37, 379-403. doi:10.1207/ S15327906MBR3703_4

Fitzmaurice, G. M., Laird, N. M., \& Ware, J. H. (2004). Applied longitudinal analysis. Hoboken, NJ: Wiley.

Fleishman, A. I. (1978). A method for simulating non-normal distributions. Psychometrika, 43, 521-532. doi:10.1007/ BF02293811

Gomez, V. E., Schaalje, G. B., \& Fellingham, G. W. (2005). Performance of the Kenward-Roger method when the covariance structure is selected using AIC and BIC. Communications in Statistics - Simulation and Computation, 34, 377-392. doi:10.1081/SAC-200055719

Gurka, M. J. (2006). Selecting the best linear mixed model under REML. American Statistician, 60, 19-26. doi:10.1198/ 000313006 X90396

Gurka, M. J., \& Edwards, L. J. (2008). Mixed models. In C. R. Rao, J. P. Miller, \& D. C. Rao (Eds.), Handbook of statistics, vol 27, epidemiological and medical statistics (pp. 253-280). New York, NY: Elsevier.

Hannan, E. J., \& Quinn, B. G. (1979). The determination of the order of an autoregression. Journal of the Royal Statistical Society, Series B, 41, 190-195.

Harville, D. A. (1974). Bayesian inference for variance components using only error contrasts. Biometrika, 61, 383-385. doi:10.1093/ biomet/61.2.383

Hurvich, C. M., \& Tsai, C. L. (1989). Regression and time series model selection in small samples. Biometrika, 76, 297-307. doi: $10.1093 /$ biomet $/ 76.2 .297$

Jennrich, R. I., \& Schluchter, M. D. (1986). Unbalanced repeatedmeasures models with structured covariance matrices. Biometrics, 42, 805-820.

Jiang, J., \& Rao, J. S. (2003). Consistent procedures for mixed linear model selection. Sankhya, 65, 23-42.

Kass, R. E., \& Raftery, A. E. (1995). Bayes factors. Journal of the American Statistical Association, 90, 773-795.

Keselman, H. J., Algina, J., Kowalchuk, R. K., \& Wolfinger, R. D. (1998). A comparison of two approaches for selecting covariance structures in the analysis of repeated measurements. Communications in Statistics-Simulation and Computation, 27, 591-604. doi:10.1080/03610919808813497

Keselman, H. J., Huberty, C. J., Lix, L. M., Olejnik, S., Cribbie, R., Donahue, B.,Kowalchuk, R. K., Lowman, L. L., Petoskey, M. D., Keselman, J. C., \& Levin, J. R.(1998). Statistical practices of educational researchers: An analysis of their ANOVA, MANOVA, 
and ANCOVA analyses. Review of Educational Research, 68, 350 386. doi:10.3102/00346543068003350

Kitagawa, G., \& Konishi, S. (2010). Bias and variance reduction techniques for bootstrap information criteria. Annals of the Institute of Statistical Mathematics, 62, 209-234. doi:10.1007/ s10463-009-0237-1

Kleinman, K., \& Horton, N. J. (2010). SAS and R: Data management, statistical analysis, and graphics. London: Chapman \& Hall/ CRC.

Kowalchuk, R. K., Lix, L. M., \& Keselman, H. J. (1996). The analysis of repeated measures designs. Paper presented at the annual meeting of the Psychometric Society, 1996, Banff, Alberta, Canada

Laird, N. M., \& Ware, J. H. (1982). Random-effects models for longitudinal data. Biometrics, 38, 963-974.

Lee, H., \& Ghosh, S. K. (2009). Performance of information criteria for spatial models. Journal of Statistical Computation and Simulation, 79, 93-106. doi:10.1080/00949650701611143

Liang, H., Wu, H., \& Zou, G. (2008). A note on conditional AIC for linear mixed-effects models. Biometrika, 95, 773-778. doi:10.1093/biomet/asn023

Lindstrom, M. J., \& Bates, D. (1988). Newton-Raphson and EM algorithms for linear mixed-effects models for repeated-measures data. Journal of the American Statistical Association, 83, 10141022.

Littell, R. C., Milliken, G. A., Stroup, W. W., Wolfinger, R. D., \& Schabenberger, O. (2006). SAS System for mixed models (2nd ed.). Cary, NC: SAS Institute Inc.

Littell, R. C., Pendergast, J., \& Natarajan, R. (2000). Modelling covariance structure in the analysis of repeated measures data. Statistics in Medicine, 19, 1793-1819. doi:10.1002/1097-0258 (20000715

Little, R. J. A., \& Rubin, D. B. (2002). Statistical analysis with missing data (2nd ed.). New York, NY: Wiley.

McCulloch, C. E., Searle, S. R., \& Neuhaus, J. M. (2008). Generalize linear, and mixed models (2nd ed.). Hoboken, NJ: Wiley.

Micceri, T. (1989). The unicorn, the normal curve, and other improbable creatures. Psychological Bulletin, 105, 156-166. doi:10.1037/0033-2909.105.1.156

Molenberghs, G., \& Kenward, M. G. (2007). Missing data in clinical studies. New York, NY: Wiley.

Molenberghs, G., \& Verbeke, G. (2001). A review on linear mixed models for longitudinal data, possibly subject to dropout. Statistical Modelling, 1, 235-269. doi:10.1177/1471082X0100100402

Patterson, H. D., \& Thompson, R. (1971). Recovery of inter-block information when block sizes are unequal. Biometrika, 58, 545554. doi:10.1093/biomet/58.3.545
Ripley, B. E. (1987). Stochastic simulation. New York, NY: Wiley.

SAS Institute Inc. (2008). SAS/STAT ® 9.2 user's guide. Cary, NC: Author.

Schafer, J. L., \& Graham, J. W. (2002). Missing data: Our view of the state of the art. Psychological Methods, 7, 147-177. doi:10.1037/ 1082-989X.7.2.147

Schwarz, G. (1978). Estimating the dimension of a model. Annals of Statistics, 6, 461-464. doi:10.1214/aos/1176344136

Shang, J., \& Cavanaugh, J. E. (2008). Bootstrap variants of the Akaike information criterion for mixed model selection. Computational Statistics and Data Analysis, 52, 2004-2021. doi:10.1016/j.csda.2007.06.019

Siddiqui, O., Hung, H. M. J., \& O’Neill, R. (2009). MMRM vs. LOCF: A comprehensive comparison based on simulation study and 25 NDA datasets. Journal of Biopharmaceutical Statistics, 19, 227-246. doi:10.1080/10543400802609797

Singer, J. D., \& Willett, J. B. (2003). Applied longitudinal data analysis: Modeling change and event occurrence. New York, NY: Oxford University Press.

Sugiura, N. (1978). Further analysis of de data by Akaike's information criterion and the finite corrections. Communications in Statistics - Theory and Methods, 7, 13-26. doi:10.1080/ 03610927808827599

Vaida, F., \& Blanchard, S. (2005). Conditional Akaike information for mixed-effects models. Biometrika, 92, 351-370. doi:10.1093/ biomet/92.2.351

Vale, C. D., \& Maurelli, V. A. (1983). Simulating multivariate nonnormal distributions. Psychometrika, 48, 465-471. doi:10.1007/ BF02293687

Vallejo, G., Ato, M., \& Fernández, M. P. (2010). A robust approach for analyzing unbalanced factorial designs with fixed levels. Behavior Research Methods, 42, 607-617. doi:10.3758/ BRM.42.2.607

Vallejo, G., Ato, M., \& Valdés, T. (2008). Consequences of misspecifying the error covariance structure in linear mixed models for longitudinal data. Methodology: European Journal of Research Methods for the Behavioral and Social Sciences, 4, 10 21. doi:10.1027/1016-9040.12.1.10

Verbeke, G., \& Molenberghs, G. (2000). Linear mixed models for longitudinal data. New York, NY: Springer.

Wang, J., \& Schaalje, G. B. (2009). Model selection for linear mixed models using predictive criteria. Communications in Statistics Simulation and Computation, 38, 788-801. doi:10.1080/ 03610910802645362

West, B. T., Welch, K. B., \& Galecki, A. T. (2007). Linear mixed models: A practical guide using statistical software. London: Chapman \& Hall/CRC. 